\title{
Visible-Light-Promoted Direct Amination of Phenols via Oxidative Cross-Dehydrogenative Coupling Reaction
}

\author{
Yating Zhao, Binbin Huang, Chao Yang, and Wujiong Xia*
}

\begin{abstract}
State Key Lab of Urban Water Resource and Environment, School of Chemistry and Chemical Engineering, Harbin Institute of Technology, Harbin, 150080, China

E-mail: xiawj@hit.edu.cn
\end{abstract}

\section{Table of Contents}

I . General Information

II. General Procedure for CDC-amination of Phenol S2

III. Characterizations of Amination Products S3

$\begin{array}{ll}\text { IV. Gram scale reaction } & \text { S13 }\end{array}$

$\begin{array}{lr}\text { V. Control Experiments } & \text { S13 }\end{array}$

$\begin{array}{ll}\text { VI. Reference } & \text { S16 }\end{array}$

$\begin{array}{lr}\text { VII. NMR Spectra } & \text { S17 }\end{array}$ 


\section{I . General information}

Chemicals were purchased from commercial sources without further purification. Glassware was dried in oven and cooled before use. All reactions were performed with solvents dried by anhydrous $\mathrm{MgSO}_{4}$. Reactions were monitored by TLC and visualized by UV lamp (254nm) and stained with ethanolic solution of concentrated sulfuric acid or potassium permanganate. Yields generally referred to chromatographically isolated yields, unless otherwise noted.

${ }^{1} \mathrm{H}$ NMR (400MHz) and ${ }^{13} \mathrm{C}$ NMR (100 MHz) spectra are recorded on a Bruker AV-400 spectrometer in $\mathrm{CDCl}_{3}$ or $\mathrm{DMSO}-\mathrm{d}_{6}$. For ${ }^{1} \mathrm{H}$ NMR $(400 \mathrm{MHz}), \mathrm{CDCl}_{3}(\delta=7.26 \mathrm{ppm})$ and DMSO- $\mathrm{d}_{6}(\delta=2.5 \mathrm{ppm})$ serverd as internal standard and data are reported as follows: chemical shift (in ppm), multiplicity $(\mathrm{s}=$ singlet, $\mathrm{d}=$ doublet, $\mathrm{t}=$ triplet, $\mathrm{q}=$ quartet, $\mathrm{m}=$ multiplet, $\mathrm{br}=$ broad), coupling constant (in Hz), and integration. For ${ }^{13} \mathrm{C}$ NMR (100 MHz), $\mathrm{CDCl}_{3}(\delta=77.25$ ppm $)$ and $\mathrm{DMSO}_{6}(\delta=39.46 \mathrm{ppm})$ was used as internal standard. GC-MS analysis was performed on 7890A-5975C/Agilent. HR-MS spectra were recorded on a Bruker Esquire LC mass spectrometer using electrospray ionization.

Starting materials for CDC-amination were generally commercially available. The TMS-protected phenols were prepared according to previous literature. ${ }^{[1]} 1-(10 H$-phenothiazin-2-yl)ethanol, used for generation of $\mathbf{3 p}$ in $\mathrm{CDC}$-amination, was prepared by the classical $\mathrm{NaBH}_{4}$ reduction with commercial 1-(10H-phenothiazin-2-yl)ethanone.

\section{General Procedure for CDC-amination of Phenol}

A typical procedure is as follows, unless otherwise noted. Phenothiazine $(0.4 \mathrm{mmol})$, phenol (0.8 mmol) or TMS-protected phenol $(0.8 \mathrm{mmol})$ and $\mathrm{K}_{2} \mathrm{~S}_{2} \mathrm{O}_{8}(1.2 \mathrm{mmol})$ were combined in dried flask. $8 \mathrm{ml} \mathrm{MeCN}$ dried with anhydrous $\mathrm{MgSO}_{4}$ was added via syringe. The flask was capped with a rubber that pierced through by a needle, and then exposed to $8 \mathrm{~W}$ blue LED strips for irradiation in air. After reaction, the mixture was diluted with DCM and filtered through an inch of silica gel. Then the filtrate was concentrated for purification by chromatography on silica gel to afford product. 


\section{. Characterizations of Amination Products}<smiles>COc1ccc(O)c(N2c3ccccc3Sc3ccccc32)c1</smiles>

Compound 3a. ${ }^{[2]}$ Isolated yield: $32 \%$, white solid. Prepared following the general procedure using phenothiazine ( $80 \mathrm{mg}, 0.4 \mathrm{mmol}$ ), 4-methoxyphenol (99 mg, $0.8 \mathrm{mmol}$ ) and $\mathrm{K}_{2} \mathrm{~S}_{2} \mathrm{O}_{8}(324 \mathrm{mg}, 1.2$ $\mathrm{mmol})$ in $\mathrm{MeCN}(8.0 \mathrm{ml})$. The reaction mixture was stirred open to air for $2 \mathrm{~h}$. The crude product was purified by flash column chromatography (petroleum: EtOAc, 5:1) to provide the title compound with $41 \mathrm{mg}$. When 4-methoxyphenol was replaced with the TMS-protected one (156 $\mathrm{mg}, 0.8 \mathrm{mmol})$, the title compound was obtained in $30 \%$ yield $(38 \mathrm{mg}) .{ }^{1} \mathrm{H}$ NMR $(600 \mathrm{MHz}$, DMSO): $\delta 9.47(\mathrm{~s}, 1 \mathrm{H}), 7.07(\mathrm{dd}, \mathrm{J}=8.9,2.1 \mathrm{~Hz}, 1 \mathrm{H}), 6.99(\mathrm{dd}, \mathrm{J}=4.3,2.7 \mathrm{~Hz}, 3 \mathrm{H}), 6.93-6.88$ $(\mathrm{m}, 2 \mathrm{H}), 6.82-6.78(\mathrm{~m}, 3 \mathrm{H}), 6.09(\mathrm{~d}, \mathrm{~J}=8.2 \mathrm{~Hz}, 2 \mathrm{H}), 3.70(\mathrm{~s}, 3 \mathrm{H}) ;{ }^{13} \mathrm{C}$ NMR (151 MHz, DMSO): $\delta 153.46,149.23,142.63,127.24,126.39,126.14,122.17,118.20,117.75,116.17,115.33,115.31$, 55.49; IR (neat, $\mathrm{cm}^{-1}$ ): v: 3478, 1500, 1461, 1306, 813, 745; GC-MS (EI): 321.2, 289.1, 273.1, 257.1, 198.1, 167.1; HRMS $(\mathrm{m} / \mathrm{z})$ : $[\mathrm{M}]^{+}$calculated for $\mathrm{C}_{19} \mathrm{H}_{15} \mathrm{NO}_{2} \mathrm{~S}, 321.0823$; found 321.0828.<smiles>Oc1cc2c(cc1N1c3ccccc3Sc3ccccc31)OCO2</smiles>

Compound 3b. Isolated yield: 97\%, white solid. Prepared following the general procedure using phenothiazine ( $80 \mathrm{mg}, 0.4 \mathrm{mmol}$ ), sesamol (110 mg, $0.8 \mathrm{mmol})$ and $\mathrm{K}_{2} \mathrm{~S}_{2} \mathrm{O}_{8}(324 \mathrm{mg}, 1.2 \mathrm{mmol})$ in $\mathrm{MeCN}(8.0 \mathrm{ml})$. The reaction mixture was stirred open to air for $1.5 \mathrm{~h}$. The crude product was purified by flash column chromatography (petroleum: EtOAc, 5:1) to provide the title compound with $129 \mathrm{mg}$. When phenol was replaced with the TMS-protected one $(168 \mathrm{mg}, 0.8 \mathrm{mmol})$, the title compound was obtained in 95\% yield (127 mg). ${ }^{1} \mathrm{H}$ NMR (400 MHz, DMSO): $\delta 9.61(\mathrm{~s}, 1 \mathrm{H})$, $6.95(\mathrm{dd}, J=7.5,1.4 \mathrm{~Hz}, 2 \mathrm{H}), 6.93-6.86(\mathrm{~m}, 2 \mathrm{H}), 6.81(\mathrm{~s}, 1 \mathrm{H}), 6.78(\mathrm{td}, J=7.4,1.1 \mathrm{~Hz}, 2 \mathrm{H})$, $6.71(\mathrm{~s}, 1 \mathrm{H}), 6.17-6.10(\mathrm{~m}, 2 \mathrm{H}), 6.04(\mathrm{~s}, 2 \mathrm{H}) ;{ }^{13} \mathrm{C}$ NMR (151 MHz, DMSO): $\delta 150.33,147.86$, 142.80, 140.87, 127.19, 126.07, 122.08, 118.17, 117.84, 115.29, 110.18, 101.50, 98.58; IR (neat, $\left.\mathrm{cm}^{-1}\right): v: 3413,1460,1305,1217,1034,745$; GC-MS (EI): 335.1, 301.1, 276.1, 244.1, 198.1, 167.2; HRMS (m/z): [M] calculated for $\mathrm{C}_{19} \mathrm{H}_{13} \mathrm{NO}_{3} \mathrm{~S}, 335.0616$; found 335.0613. 
<smiles>COc1cc(O)c(N2c3ccccc3Sc3ccccc32)cc1OC</smiles>

Compound 3c. Isolated yield: $95 \%$, dark foamy solid. Prepared following the general procedure using phenothiazine ( $80 \mathrm{mg}, 0.4 \mathrm{mmol})$, 3,4-dimethoxyphenol $\left(123 \mathrm{mg}, 0.8 \mathrm{mmol}\right.$ ) and $\mathrm{K}_{2} \mathrm{~S}_{2} \mathrm{O}_{8}$ (324 mg, $1.2 \mathrm{mmol}$ ) in MeCN (8.0 ml). The reaction mixture was stirred open to air for $1.5 \mathrm{~h}$. The crude product was purified by flash column chromatography (petroleum: EtOAc, 5:1) to provide the title compound with $133 \mathrm{mg} .{ }^{1} \mathrm{H}$ NMR (600 MHz, DMSO): $\delta 9.48$ (s, $1 \mathrm{H}$ ), 6.97 (dd, $J=7.5$, $1.3 \mathrm{~Hz}, 2 \mathrm{H}), 6.93-6.86(\mathrm{~m}, 2 \mathrm{H}), 6.78(\mathrm{dd}, J=10.7,4.1 \mathrm{~Hz}, 2 \mathrm{H}), 6.76(\mathrm{~s}, 1 \mathrm{H}), 6.74(\mathrm{~s}, 1 \mathrm{H}), 6.16-$ 6.09 (m, 2H), 3.81 (s, 3H), 3.67 (s, 3H); ${ }^{13} \mathrm{C}$ NMR (151 MHz, DMSO): $\delta$ 149.69, 149.36, 143.02, $142.90,127.20,126.06,122.03,118.25,116.94,115.43,113.87,101.47,56.06,55.44$; IR (neat, $\left.\mathrm{cm}^{-1}\right): v: 3395,1507,1461,1234,914,744$; GC-MS (EI): 351.2, 335.1, 319.1, 276.1, 198.1, 167.1; HRMS $(m / z):[\mathrm{M}+\mathrm{H}]^{+}$calculated for $\mathrm{C}_{20} \mathrm{H}_{18} \mathrm{NO}_{3} \mathrm{~S}, 352.1007$; found 352.1009 .<smiles>Cc1cc(O)c(N2c3ccccc3Sc3ccccc32)cc1C</smiles>

Compound 3d. Isolated yield: $61 \%$, white sticky solid. Prepared following the general procedure using phenothiazine ( $80 \mathrm{mg}, 0.4 \mathrm{mmol}$ ), 3,4-dimethylphenol (97 mg, $0.8 \mathrm{mmol}$ ) and $\mathrm{K}_{2} \mathrm{~S}_{2} \mathrm{O}_{8}(324$ $\mathrm{mg}, 1.2 \mathrm{mmol})$ in $\mathrm{MeCN}(8.0 \mathrm{ml})$. The reaction mixture was stirred open to air for $2 \mathrm{~h}$. The crude product was purified by flash column chromatography (petroleum: DCM, 2:1) to provide the title compound with $77 \mathrm{mg}$. ${ }^{1} \mathrm{H}$ NMR (400 MHz, DMSO): $\delta 9.55(\mathrm{~s}, 1 \mathrm{H}), 7.00-6.93(\mathrm{~m}, 3 \mathrm{H}), 6.91(\mathrm{~s}, 1 \mathrm{H})$, $6.90-6.84(\mathrm{~m}, 2 \mathrm{H}), 6.77(\mathrm{t}, J=7.0 \mathrm{~Hz}, 2 \mathrm{H}), 6.08(\mathrm{~d}, J=8.2 \mathrm{~Hz}, 2 \mathrm{H}), 2.23(\mathrm{~s}, 3 \mathrm{H}), 2.15(\mathrm{~s}, 3 \mathrm{H}) ;{ }^{13} \mathrm{C}$ NMR (151 MHz, DMSO): $\delta$ 153.12, 142.96, 138.23, 131.39, 128.67, 127.16, 126.07, 123.62, 122.00, 118.27, 118.20, 115.44, 19.38, 18.26; IR (neat, $\mathrm{cm}^{-1}$ ): v: 3410, 2994, 1495, 1460, 1306, 744; GC-MS (EI): $319.2,303.1,286.1,271.1,198.1,167.1$; $\operatorname{HRMS}(\mathrm{m} / \mathrm{z}):[\mathrm{M}]^{+}$calculated for $\mathrm{C}_{20} \mathrm{H}_{17} \mathrm{NOS}$, 319.1031; found 319.1028.<smiles>CCOc1ccc(O)c(N2c3ccccc3Sc3ccccc32)c1</smiles>

Compound 3e. Isolated yield: $41 \%$, white solid. Prepared following the general procedure using phenothiazine ( $80 \mathrm{mg}, 0.4 \mathrm{mmol})$, 4-ethoxyphenol (110 mg, $0.8 \mathrm{mmol})$ and $\mathrm{K}_{2} \mathrm{~S}_{2} \mathrm{O}_{8}(324 \mathrm{mg}, 1.2$ $\mathrm{mmol})$ in $\mathrm{MeCN}(8.0 \mathrm{ml})$. The reaction mixture was stirred open to air for $2 \mathrm{~h}$. The crude product 
was purified by flash column chromatography (petroleum: EtOAc, 5:1) to provide the title compound with $54 \mathrm{mg} .{ }^{1} \mathrm{H}$ NMR (400 MHz, DMSO): $\delta 9.43$ (s, 1H), 7.05 (d, $\left.J=8.9 \mathrm{~Hz}, 1 \mathrm{H}\right)$, $7.00-6.94(\mathrm{~m}, 3 \mathrm{H}), 6.93-6.86(\mathrm{~m}, 2 \mathrm{H}), 6.83-6.74(\mathrm{~m}, 3 \mathrm{H}), 6.12-6.04(\mathrm{~m}, 2 \mathrm{H}), 3.95(\mathrm{q}, J=$ $6.9 \mathrm{~Hz}, 2 \mathrm{H}), 1.27$ (t, $J=6.9 \mathrm{~Hz}, 3 \mathrm{H}) ;{ }^{13} \mathrm{C}$ NMR $(151 \mathrm{MHz}, \mathrm{DMSO}): \delta 152.68,149.10,142.62$, $127.22,126.32,126.12,122.14,118.14,117.77,116.72,115.96,115.29,63.48,14.70$; IR (neat, $\mathrm{cm}^{-1}$ ): v: 3386, 2913, 1500, 1460, 1231, 743; GC-MS (EI): 335.2, 306.1, 289.1, 273.1, 198.1, 167.1; HRMS $(\mathrm{m} / \mathrm{z}):[\mathrm{M}]^{+}$calculated for $\mathrm{C}_{20} \mathrm{H}_{17} \mathrm{NO}_{2} \mathrm{~S}, 335.0980$; found 335.0987.<smiles>Oc1ccc(OCc2ccccc2)c(N2c3ccccc3Sc3ccccc32)c1</smiles>

Compound 3f. Isolated yield: 45\%, white solid. Prepared following the general procedure using phenothiazine ( $80 \mathrm{mg}, 0.4 \mathrm{mmol}$ ), 4-(benzyloxy)phenol (160 mg, $0.8 \mathrm{mmol})$ and $\mathrm{K}_{2} \mathrm{~S}_{2} \mathrm{O}_{8}(324 \mathrm{mg}$, $1.2 \mathrm{mmol})$ in $\mathrm{MeCN}(8.0 \mathrm{ml})$. The reaction mixture was stirred open to air for $3 \mathrm{~h}$. The crude product was purified by flash column chromatography (petroleum: EtOAc, 5:1) to provide the title compound with $71 \mathrm{mg}$. When phenol was replaced with the TMS-protected one (217 mg, 0.8 $\mathrm{mmol})$, the title compound was obtained in $34 \%$ yield $(53 \mathrm{mg}) .{ }^{1} \mathrm{H}$ NMR (400 MHz, DMSO): $\delta$ 9.48 (s, 1H), 7.42 (d, $J=7.0 \mathrm{~Hz}, 2 \mathrm{H}), 7.36$ (t, $J=7.3 \mathrm{~Hz}, 2 \mathrm{H}), 7.32$ (d, $J=7.0 \mathrm{~Hz}, 1 \mathrm{H}), 7.06$ (s, 2H), $6.98(\mathrm{dd}, J=7.5,1.4 \mathrm{~Hz}, 2 \mathrm{H}), 6.92-6.85(\mathrm{~m}, 3 \mathrm{H}), 6.79(\mathrm{t}, J=7.0 \mathrm{~Hz}, 2 \mathrm{H}), 6.07$ (d, $J=7.6$ $\mathrm{Hz}, 2 \mathrm{H}), 5.03$ (s, 2H); ${ }^{13} \mathrm{C}$ NMR (151 MHz, DMSO) $\delta 152.41,149.40,142.62,137.09,128.32$, $127.78,127.73,127.21,126.30,126.13,122.16,118.22$, 117.81, 117.35, 116.61, 115.32, 69.86; IR (neat, $\mathrm{cm}^{-1}$ ): $v: 3408,3062,1500,1462,815,746$; GC-MS (EI): 397.2, 306.1, 289.1, 273.1, 199.1, 91.2; HRMS $(\mathrm{m} / \mathrm{z})$ : $[\mathrm{M}+\mathrm{K}]^{+}$calculated for $\mathrm{C}_{25} \mathrm{H}_{19} \mathrm{KNO}_{2} \mathrm{~S}, 436.0774$; found 436.0772 .<smiles>COc1cc(Cl)c(O)c(N2c3ccccc3Sc3ccccc32)c1</smiles>

Compound 3g. Isolated yield: 67\%, white foamy to sticky solid. Prepared following the general procedure using phenothiazine ( $80 \mathrm{mg}, 0.4 \mathrm{mmol}), 2$-chloro-4-methoxyphenol (126 mg, $0.8 \mathrm{mmol}$ ) and $\mathrm{K}_{2} \mathrm{~S}_{2} \mathrm{O}_{8}$ (324 mg, $\left.1.2 \mathrm{mmol}\right)$ in $\mathrm{MeCN}(8.0 \mathrm{ml})$. The reaction mixture was stirred open to air for $2 \mathrm{~h}$. The crude product was purified by flash column chromatography (petroleum: DCM, 3:1) to provide the title compound with $95 \mathrm{mg}$. When phenol was replaced with the TMS-protected one (184 mg, $0.8 \mathrm{mmol}$ ), the title compound was obtained in $51 \%$ yield $(72 \mathrm{mg}) .{ }^{1} \mathrm{H}$ NMR (400 MHz, DMSO): $\delta 9.48(\mathrm{~s}, 1 \mathrm{H}), 7.21(\mathrm{~d}, J=3.0 \mathrm{~Hz}, 1 \mathrm{H}), 6.99$ (s, 1H), 6.97 (d, $J=1.3 \mathrm{~Hz}, 1 \mathrm{H}), 6.91$ (dd, $J$ $=10.9,4.5 \mathrm{~Hz}, 2 \mathrm{H}), 6.85(\mathrm{~d}, J=3.0 \mathrm{~Hz}, 1 \mathrm{H}), 6.80(\mathrm{t}, J=7.4 \mathrm{~Hz}, 2 \mathrm{H}), 6.03(\mathrm{~d}, J=8.1 \mathrm{~Hz}, 2 \mathrm{H})$, 3.73 (s, 3H); ${ }^{13} \mathrm{C}$ NMR (151 MHz, DMSO) $\delta 153.33,145.59,142.10,128.94,127.32,126.15$, $122.99,122.36,118.26,116.29,115.36,115.17,114.27,55.85$; IR (neat, $\mathrm{cm}^{-1}$ ): $v: 3484,1581$, 1461, 1229, 1041, 758; GC-MS (EI): 355.2, 323.1, 287.1, 198.1, 167.1, 152.4; HRMS (m/z): 
$[\mathrm{M}+\mathrm{H}]^{+}$calculated for $\mathrm{C}_{19} \mathrm{H}_{15} \mathrm{ClNO}_{2} \mathrm{~S}, 356.0512$; found 356.0519 .<smiles>COc1cccc(N2c3ccccc3Sc3ccccc32)c1O</smiles>

Compound 3h. Isolated yield: $69 \%$, white foamy solid. Prepared following the general procedure using phenothiazine ( $80 \mathrm{mg}, 0.4 \mathrm{mmol}$ ), 2-methoxyphenol $\left(99 \mathrm{mg}, 0.8 \mathrm{mmol}\right.$ ) and $\mathrm{K}_{2} \mathrm{~S}_{2} \mathrm{O}_{8}(324$ $\mathrm{mg}, 1.2 \mathrm{mmol})$ in $\mathrm{MeCN}(8.0 \mathrm{ml})$. The reaction mixture was stirred open to air for $2 \mathrm{~h}$. The crude product was purified by flash column chromatography (petroleum: EA, 5:1) to provide the title compound with $88 \mathrm{mg} .{ }^{1} \mathrm{H}$ NMR (400 MHz, DMSO): $\delta 9.43$ (s, 1H), $7.05-6.98$ (m, 3H), $6.94-$ $6.87(\mathrm{~m}, 3 \mathrm{H}), 6.81(\mathrm{t}, J=7.1 \mathrm{~Hz}, 3 \mathrm{H}), 6.22(\mathrm{~d}, J=8.2 \mathrm{~Hz}, 2 \mathrm{H}), 3.77(\mathrm{~s}, 3 \mathrm{H}) ;{ }^{13} \mathrm{C} \mathrm{NMR}(151 \mathrm{MHz}$, DMSO): $\delta$ 149.37, 146.60, 144.04, 130.93, 127.22, 126.34, 122.92, 122.29, 118.37, 116.67, 115.53, 113.97, 55.78; IR (neat, $\mathrm{cm}^{-1}$ ): $v: 3404,1585,1507,1460,1305$, 746; GC-MS (EI): 321.2, 306.1, 278.1, 260.1, 198.1, 130.4; HRMS (m/z): $[\mathrm{M}+\mathrm{Na}]^{+}$calculated for $\mathrm{C}_{19} \mathrm{H}_{15} \mathrm{NaNO}_{2} \mathrm{~S}, 344.0721$; found 344.0725 .<smiles>COc1cc(C)cc(N2c3ccccc3Sc3ccccc32)c1O</smiles>

Compound 3i. Isolated yield: 52\%, white foamy solid. Prepared following the general procedure using phenothiazine (40 mg, $0.2 \mathrm{mmol}), 2$-methoxy-4-methylphenol $(55 \mathrm{mg}, 0.4 \mathrm{mmol})$ and $\mathrm{K}_{2} \mathrm{~S}_{2} \mathrm{O}_{8}(162 \mathrm{mg}, 0.6 \mathrm{mmol})$ in $\mathrm{MeCN}(4.0 \mathrm{ml})$. The reaction mixture was stirred open to air for 2 $\mathrm{h}$. The crude product was purified by flash column chromatography (petroleum: DCM, 2:1) to provide the title compound with $34 \mathrm{mg}$. ${ }^{1} \mathrm{H}$ NMR (400 MHz, DMSO): $\delta 8.93$ (s, 1H), $7.00-6.94$ (m, 3H), $6.91-6.84$ (m, 2H), 6.77 (td, $J=7.4,1.0 \mathrm{~Hz}, 2 \mathrm{H}), 6.63$ (s, 1H), 6.07 (dd, $J=8.2,0.8 \mathrm{~Hz}$, 2H), 3.87 (s, 3H), 2.27 (s, 3H); ${ }^{13} \mathrm{C}$ NMR (151 MHz, DMSO): $\delta 148.85,142.62,142.39,129.49$, $127.19,126.18,126.09,122.18,122.07,118.20,115.36,113.07,55.91,20.51$; IR (neat, $\left.\mathrm{cm}^{-1}\right): v$ : 3403, 1584, 1500, 1460, 1234, 742; GC-MS (EI): 335.1, 319.1, 303.1, 287.1, 198.0, 167.1; HRMS $(\mathrm{m} / \mathrm{z}):[\mathrm{M}+\mathrm{H}]^{+}$calculated for $\mathrm{C}_{20} \mathrm{H}_{18} \mathrm{NO}_{2} \mathrm{~S}, 336.1058$; found 336.1056 .<smiles>Oc1ccc2ccccc2c1N1c2ccccc2Sc2ccccc21</smiles>

Compound 3j. Isolated yield: 93\%, white solid. Prepared following the general procedure using phenothiazine ( $80 \mathrm{mg}, 0.4 \mathrm{mmol}$ ), 2-naphthol (115 mg, $0.8 \mathrm{mmol})$ and $\mathrm{K}_{2} \mathrm{~S}_{2} \mathrm{O}_{8}(324 \mathrm{mg}, 1.2 \mathrm{mmol}$ ) 
in $\mathrm{MeCN}(8.0 \mathrm{ml})$. The reaction mixture was stirred open to air for $2 \mathrm{~h}$. The crude product was purified by flash column chromatography (petroleum: EA, 5:1) to provide the title compound with $126 \mathrm{mg}$. When phenol was replaced with the TMS-protected one (172 $\mathrm{mg}, 0.8 \mathrm{mmol})$, the title compound was obtained in $86 \%$ yield $(117 \mathrm{mg}) .{ }^{1} \mathrm{H}$ NMR (400 MHz, DMSO) $\delta 10.34(\mathrm{~s}, 1 \mathrm{H})$, $8.00(\mathrm{~d}, J=8.9 \mathrm{~Hz}, 1 \mathrm{H}), 7.97-7.91(\mathrm{~m}, 2 \mathrm{H}), 7.45(\mathrm{t}, J=8.6 \mathrm{~Hz}, 2 \mathrm{H}), 7.35(\mathrm{t}, J=7.5 \mathrm{~Hz}, 1 \mathrm{H})$, $7.03(\mathrm{~d}, J=7.2 \mathrm{~Hz}, 2 \mathrm{H}), 6.86-6.74(\mathrm{~m}, 4 \mathrm{H}), 6.01(\mathrm{~d}, J=7.8 \mathrm{~Hz}, 2 \mathrm{H}) ;{ }^{13} \mathrm{C}$ NMR $(151 \mathrm{MHz}$, DMSO): $\delta 153.84,142.13,131.60,130.30,129.15,128.44,127.60,127.36,126.26,123.52$, 122.36, 120.92, 118.91, 117.84, 115.30; IR (neat, $\mathrm{cm}^{-1}$ ): v: 3415, 1598, 1461, 1307, 817, 743; GC-MS (EI): 341.2, 280.1，198.1，167.1，144.2, 115.1; HRMS $(\mathrm{m} / \mathrm{z}):[\mathrm{M}]^{+}$calculated for $\mathrm{C}_{22} \mathrm{H}_{15} \mathrm{NOS}$, 341.0874; found 341.0877 .<smiles>Oc1ccc2c(cccc3c4ccccc4sc4ccccc4n2-3)c1</smiles>

Compound 3k. Isolated yield: $89 \%$, white fluffy solid. Prepared following the general procedure using phenothiazine ( $80 \mathrm{mg}, 0.4 \mathrm{mmol}), 2,6$-dihydroxynaphthalene (128 $\mathrm{mg}, 0.8 \mathrm{mmol})$ and $\mathrm{K}_{2} \mathrm{~S}_{2} \mathrm{O}_{8}$ (324 mg, $\left.1.2 \mathrm{mmol}\right)$ in $\mathrm{MeCN}(8.0 \mathrm{ml})$. The reaction mixture was stirred open to air for 2 h. The crude product was purified by flash column chromatography (petroleum: EA, 5:1) to provide the title compound with $126 \mathrm{mg} .{ }^{1} \mathrm{H}$ NMR (400 MHz, DMSO): $\delta 10.11(\mathrm{~s}, 1 \mathrm{H}), 9.75$ (s, $1 \mathrm{H}), 7.82(\mathrm{~d}, J=8.9 \mathrm{~Hz}, 1 \mathrm{H}), 7.76(\mathrm{~d}, J=8.8 \mathrm{~Hz}, 1 \mathrm{H}), 7.16$ (d, $J=8.8 \mathrm{~Hz}, 2 \mathrm{H}), 6.99$ (dd, $J=7.2$, $1.9 \mathrm{~Hz}, 2 \mathrm{H}), 6.88(\mathrm{dd}, J=8.7,2.4 \mathrm{~Hz}, 1 \mathrm{H}), 6.78(\mathrm{pd}, J=7.3,1.7 \mathrm{~Hz}, 4 \mathrm{H}), 5.97$ (dd, $J=7.8,1.6$ $\mathrm{Hz}, 2 \mathrm{H}) ;{ }^{13} \mathrm{C}$ NMR $(151 \mathrm{MHz}$, DMSO): $\delta 156.84,153.91,141.89,133.49,130.23,129.97,127.32$, 126.12, 123.74, 122.17, 118.50, 116.26, 115.90, 115.21, 115.08, 102.75; IR (neat, $\mathrm{cm}^{-1}$ ): $v: 3425$, 3241, 1683, 1460, 828, 745; GC-MS (EI): 357.1, 331.0, 315.1, 253.0, 199.0, 167.1; HRMS (m/z): $[\mathrm{M}]^{+}$calculated for $\mathrm{C}_{22} \mathrm{H}_{15} \mathrm{NO}_{2} \mathrm{~S}, 357.0823$; found 357.0828 .<smiles>Oc1ccc2cc(Br)ccc2c1N1c2ccccc2Sc2ccccc21</smiles>

Compound 31. Isolated yield: 70\%, white fluffy solid. Prepared following the general procedure using phenothiazine (40 mg, $0.2 \mathrm{mmol}$ ), 6-bromo-2-naphthol (89 mg, $0.4 \mathrm{mmol})$ and $\mathrm{K}_{2} \mathrm{~S}_{2} \mathrm{O}_{8}(162$ $\mathrm{mg}, 0.6 \mathrm{mmol})$ in $\mathrm{MeCN}(4.0 \mathrm{ml})$. The reaction mixture was stirred open to air for $2 \mathrm{~h}$. The crude product was purified by flash column chromatography (petroleum: DCM, 2:1) to provide the title compound with $58 \mathrm{mg} .{ }^{1} \mathrm{H}$ NMR (400 MHz, $\mathrm{CDCl}_{3}$ ): $\delta 8.07$ (s, $\left.1 \mathrm{H}\right), 7.84(\mathrm{~d}, J=9.0 \mathrm{~Hz}, 1 \mathrm{H}), 7.60$ $(\mathrm{d}, J=9.0 \mathrm{~Hz}, 1 \mathrm{H}), 7.48(\mathrm{~d}, J=8.2 \mathrm{~Hz}, 1 \mathrm{H}), 7.43(\mathrm{~d}, J=9.0 \mathrm{~Hz}, 1 \mathrm{H}), 7.11(\mathrm{~d}, J=7.4 \mathrm{~Hz}, 2 \mathrm{H})$, $6.88(\mathrm{t}, J=7.4 \mathrm{~Hz}, 2 \mathrm{H}), 6.80(\mathrm{t}, J=7.7 \mathrm{~Hz}, 2 \mathrm{H}), 6.38(\mathrm{~s}, 1 \mathrm{H}), 6.10(\mathrm{~d}, J=8.1 \mathrm{~Hz}, 2 \mathrm{H}) ;{ }^{13} \mathrm{C} \mathrm{NMR}$ $\left(151 \mathrm{MHz}, \mathrm{CDCl}_{3}\right): \delta 152.16,142.35,130.93,130.91,130.73,129.82,127.50,127.00,124.82$, 
123.68, 121.24, 119.35, 119.14, 117.89, 116.05; IR (neat, $\mathrm{cm}^{-1}$ ): v: 3520, 1636, 1460, 1262, 747, 613; GC-MS (EI): 421.0, 307.1, 278.1, 222.0, 198.0, 167.1; HRMS $(\mathrm{m} / \mathrm{z})$ : $[\mathrm{M}]^{+}$calculated for $\mathrm{C}_{22} \mathrm{H}_{14} \mathrm{BrNOS}$, 418.9979; found 418.9983 .<smiles>Oc1c(N2c3ccccc3Sc3ccccc32)ccc2ccccc12</smiles>

Compound 3m. Isolated yield: $52 \%$, brown foamy solid. Prepared following the general procedure using phenothiazine ( $80 \mathrm{mg}, 0.4 \mathrm{mmol}), 1$-naphthol (115 mg, $0.8 \mathrm{mmol}$ ) and $\mathrm{K}_{2} \mathrm{~S}_{2} \mathrm{O}_{8}$ (324 mg, $1.2 \mathrm{mmol})$ in $\mathrm{MeCN}(8.0 \mathrm{ml})$. The reaction mixture was stirred open to air for $12 \mathrm{~h}$. The crude product was purified by flash column chromatography (petroleum: EA, 5:1) to provide the title compound with $70 \mathrm{mg} .{ }^{1} \mathrm{H}$ NMR $\left(400 \mathrm{MHz}, \mathrm{CDCl}_{3}\right): \delta 8.31(\mathrm{~d}, J=8.3 \mathrm{~Hz}, 1 \mathrm{H}), 7.99(\mathrm{~d}, J=$ $8.3 \mathrm{~Hz}, 1 \mathrm{H}), 7.54$ (t, $J=7.2 \mathrm{~Hz}, 1 \mathrm{H}), 7.50-7.41(\mathrm{~m}, 2 \mathrm{H}), 7.05-6.93(\mathrm{~m}, 3 \mathrm{H}), 6.73$ (dtd, $J=17.0$, 7.4, $1.3 \mathrm{~Hz}, 4 \mathrm{H}), 6.05$ (dd, $J=7.9,1.0 \mathrm{~Hz}, 3 \mathrm{H}) ;{ }^{13} \mathrm{C} \mathrm{NMR}\left(151 \mathrm{MHz}, \mathrm{CDCl}_{3}\right): \delta 152.10,127.78$, $126.93,126.48,126.02,123.72,122.75,122.32,115.81,108.93,77.28,77.07,76.86$; IR (neat, $\left.\mathrm{cm}^{-1}\right): v: 3398,3061,1589,1461,1305,744$; GC-MS (EI): 341.1, 308.1, 280.1, 199.1, 167.1, 115.1; HRMS $(\mathrm{m} / \mathrm{z})$ : $[\mathrm{M}]^{+}$calculated for $\mathrm{C}_{22} \mathrm{H}_{15} \mathrm{NOS}, 341.0874$; found 341.0873 .<smiles>COc1cc(O)c(N2c3ccccc3Oc3ccccc32)cc1OC</smiles>

Compound 3n. Isolated yield: $96 \%$, white solid. Prepared following the general procedure using phenoxazine (73 mg, $0.4 \mathrm{mmol}$ ), 3,4-dimethoxyphenol (123 mg, $0.8 \mathrm{mmol})$ and $\mathrm{K}_{2} \mathrm{~S}_{2} \mathrm{O}_{8}(324 \mathrm{mg}$, $1.2 \mathrm{mmol})$ in $\mathrm{MeCN}(8.0 \mathrm{ml})$. The reaction mixture was stirred open to air for $12 \mathrm{~h}$. The crude product was purified by flash column chromatography (petroleum: EA, 5:1) to provide the title compound with $128 \mathrm{mg} .{ }^{1} \mathrm{H}$ NMR (600 MHz, DMSO): $\delta 9.36$ (s, 1H), 6.77 (s, 1H), $6.70(\mathrm{~s}, 1 \mathrm{H})$, $6.63(\mathrm{ddd}, J=18.7,11.7,7.5 \mathrm{~Hz}, 6 \mathrm{H}), 5.88(\mathrm{~d}, J=7.6 \mathrm{~Hz}, 2 \mathrm{H}), 3.78(\mathrm{~s}, 3 \mathrm{H}), 3.66(\mathrm{~s}, 3 \mathrm{H}) ;{ }^{13} \mathrm{C}$ NMR (151 MHz, DMSO): $\delta$ 149.76, 149.16, 143.39, 142.87, 133.70, 123.55, 120.77, 114.81, 114.04, 112.82, 101.86, 56.13, 55.41; IR (neat, $\left.\mathrm{cm}^{-1}\right): v: 3425,1588,1487,1270,1202,744$; GC-MS (EI): 335.2, 319.2, 291.1, 248.1, 220.1, 182.1; HRMS $(\mathrm{m} / \mathrm{z}):[\mathrm{M}]^{+}$calculated for $\mathrm{C}_{20} \mathrm{H}_{17} \mathrm{NO}_{4}, 335.1158$; found 335.1159. 
<smiles>CCSc1ccc2c(c1)N(c1cc(OC)c(OC)cc1O)c1ccccc1S2</smiles>

Compound 3o. Isolated yield: $82 \%$, dark brown viscous oil. Prepared following the general procedure using 2-(ethylthio)-10H-phenothiazine (103 mg, $0.4 \mathrm{mmol}$ ), 3,4-dimethoxyphenol (123 $\mathrm{mg}, 0.8 \mathrm{mmol})$ and $\mathrm{K}_{2} \mathrm{~S}_{2} \mathrm{O}_{8}(324 \mathrm{mg}, 1.2 \mathrm{mmol})$ in $\mathrm{MeCN}(8.0 \mathrm{ml})$. The reaction mixture was stirred open to air for $1.5 \mathrm{~h}$. The crude product was purified by flash column chromatography (petroleum: EA, 5:1) to provide the title compound with $134 \mathrm{mg} .{ }^{1} \mathrm{H}$ NMR (400 MHz, DMSO): $\delta$ $9.53(\mathrm{~s}, 1 \mathrm{H}), 7.01-6.86(\mathrm{~m}, 3 \mathrm{H}), 6.82-6.70(\mathrm{~m}, 4 \mathrm{H}), 6.12(\mathrm{dd}, J=8.2,0.9 \mathrm{~Hz}, 1 \mathrm{H}), 6.05(\mathrm{~d}, J=$ $1.8 \mathrm{~Hz}, 1 \mathrm{H}), 3.82$ (s, 3H), 3.67 (s, 3H), 2.74 (q, $J=7.3 \mathrm{~Hz}, 2 \mathrm{H}), 1.11(\mathrm{t}, J=7.3 \mathrm{~Hz}, 3 \mathrm{H}) ;{ }^{13} \mathrm{C}$ NMR (151 MHz, DMSO): $\delta 149.81,149.30,143.35,142.93,142.73,134.75,127.25,126.49,126.11$, $122.24,121.65,118.29,116.61,115.84,115.63,115.15,113.69,101.35,56.07,55.46,26.45,14.15$; IR (neat, $\mathrm{cm}^{-1}$ ): $v: 3391,1508,1461,1385,1233,749$; GC-MS (EI): 411.2, 367.1, 335.1, 317.1, 259.1, 230.0, 198.1; HRMS (m/z): [M] $]^{+}$calculated for $\mathrm{C}_{22} \mathrm{H}_{21} \mathrm{NO}_{3} \mathrm{~S}_{2}, 411.0963$; found 411.0963.<smiles>COc1cc(O)c(N2c3ccccc3Sc3ccc(C(C)O)cc32)cc1OC</smiles>

Compound 3p. Isolated yield: $88 \%$, dark red viscous oil. Prepared following the general procedure using 1-(10H-phenothiazin-2-yl)ethanol ( $97 \mathrm{mg}, 0.4 \mathrm{mmol})$, 3,4-dimethoxyphenol (123 $\mathrm{mg}, 0.8 \mathrm{mmol})$ and $\mathrm{K}_{2} \mathrm{~S}_{2} \mathrm{O}_{8}(324 \mathrm{mg}, 1.2 \mathrm{mmol})$ in $\mathrm{MeCN}(8.0 \mathrm{ml})$. The reaction mixture was stirred open to air for $1.5 \mathrm{~h}$. The crude product was purified by flash column chromatography (petroleum: EA, 2:1) to provide the title compound with $139 \mathrm{mg} .{ }^{1} \mathrm{H}$ NMR (400 MHz, DMSO): $\delta$ $9.46(\mathrm{~s}, 1 \mathrm{H}), 6.96(\mathrm{dd}, J=7.5,1.5 \mathrm{~Hz}, 1 \mathrm{H}), 6.89$ (ddd, $J=9.6,6.8,2.4 \mathrm{~Hz}, 2 \mathrm{H}), 6.80-6.73(\mathrm{~m}$, 4H), $6.24(\mathrm{~s}, 1 \mathrm{H}), 6.10(\mathrm{~d}, J=8.2 \mathrm{~Hz}, 1 \mathrm{H}), 4.98(\mathrm{~d}, J=4.0 \mathrm{~Hz}, 1 \mathrm{H}), 4.49-4.35(\mathrm{~m}, 1 \mathrm{H}), 3.82(\mathrm{~s}$, $3 \mathrm{H}), 3.67(\mathrm{~s}, 3 \mathrm{H}), 1.14(\mathrm{~d}, J=6.4 \mathrm{~Hz}, 3 \mathrm{H}) ;{ }^{13} \mathrm{C}$ NMR (151 MHz, DMSO): $\delta 149.59,149.48$, $146.68,143.25,142.91,142.72,127.10126 .03,125.66,121.93,119.15,118.55,117.06,116.07$, $115.48,114.01,112.81,101.31,67.68,56.06,55.38,25.75$; IR (neat, $\mathrm{cm}^{-1}$ ): $v: 3415,3370,1508$, 1463, 1236, 748; GC-MS (EI): 395.2, 377.2, 335.1, 318.1, 240.1, 198.1; HRMS $(\mathrm{m} / \mathrm{z}):[\mathrm{M}+\mathrm{H}]^{+}$ calculated for $\mathrm{C}_{22} \mathrm{H}_{22} \mathrm{NO}_{4} \mathrm{~S}, 396.1270$; found 396.1270 . 
<smiles>COc1cc(O)c(N2c3ccccc3Sc3ccc(Cl)cc32)cc1OC</smiles>

Compound 3q. Isolated yield: $90 \%$, white foamy to sticky solid. Prepared following the general procedure using 2-chloro-10H-phenothiazine ( $93 \mathrm{mg}, 0.4 \mathrm{mmol})$, 3,4-dimethoxyphenol (123 mg, $0.8 \mathrm{mmol})$ and $\mathrm{K}_{2} \mathrm{~S}_{2} \mathrm{O}_{8}(324 \mathrm{mg}, 1.2 \mathrm{mmol})$ in $\mathrm{MeCN}(8.0 \mathrm{ml})$. The reaction mixture was stirred open to air for $48 \mathrm{~h}$. The crude product was purified by flash column chromatography (petroleum: EA, 5:1) to provide the title compound with $138 \mathrm{mg} .{ }^{1} \mathrm{H}$ NMR (600 MHz, DMSO): $\delta 9.63$ (s, $\left.1 \mathrm{H}\right)$, $7.02-6.95(\mathrm{~m}, 2 \mathrm{H}), 6.91(\mathrm{dd}, J=11.4,4.1 \mathrm{~Hz}, 1 \mathrm{H}), 6.80(\mathrm{ddd}, J=22.4,10.1,1.7 \mathrm{~Hz}, 4 \mathrm{H}), 6.15-$ $6.10(\mathrm{~m}, 1 \mathrm{H}), 6.06(\mathrm{~d}, J=2.2 \mathrm{~Hz}, 1 \mathrm{H}), 3.82(\mathrm{~s}, 3 \mathrm{H}), 3.68(\mathrm{~s}, 3 \mathrm{H}) ;{ }^{13} \mathrm{C}$ NMR (151 MHz, DMSO): $\delta$ 149.96, 149.12, 144.40, 143.02, 142.28, 131.67, 127.45, 127.30, 126.19, 122.67, 121.53, 118.01, 117.51, 116.26, 115.78, 114.76, 113.40, 101.45, 56.02, 55.42; IR (neat, $\left.\mathrm{cm}^{-1}\right): v: 3382,1506,1460$, 1384, 1238, 746; GC-MS (EI): 385.2, 353.1, 335.1, 317.1, 232.1, 198.1; HRMS $(m / z):[\mathrm{M}]^{+}$ calculated for $\mathrm{C}_{20} \mathrm{H}_{16} \mathrm{ClNO}_{3} \mathrm{~S}, 385.0539$; found 385.0537.<smiles>COc1cc(O)c(N2c3ccccc3Sc3ccc(Br)cc32)cc1OC</smiles>

Compound 3r. Isolated yield: 91\%, red sticky solid. Prepared following the general procedure using 2-bromo-10H-phenothiazine (55 mg, $0.2 \mathrm{mmol}$ ), 3,4-dimethoxyphenol (62 mg, $0.4 \mathrm{mmol}$ ) and $\mathrm{K}_{2} \mathrm{~S}_{2} \mathrm{O}_{8}(162 \mathrm{mg}, 0.6 \mathrm{mmol})$ in $\mathrm{MeCN}(4.0 \mathrm{ml})$. The reaction mixture was stirred open to air for $24 \mathrm{~h}$. The crude product was purified by flash column chromatography (petroleum: EA, 3:1) to provide the title compound with $78 \mathrm{mg} .{ }^{1} \mathrm{H}$ NMR (400 MHz, DMSO): $\delta 9.52$ (s, 1H), 7.17 (d, $J=$ $2.3 \mathrm{~Hz}, 1 \mathrm{H}), 7.06(\mathrm{dd}, J=8.8,2.3 \mathrm{~Hz}, 1 \mathrm{H}), 6.99-6.95(\mathrm{~m}, 1 \mathrm{H}), 6.94-6.88(\mathrm{~m}, 1 \mathrm{H}), 6.80(\mathrm{td}, J=$ 7.5, $1.0 \mathrm{~Hz}, 1 \mathrm{H}), 6.76(\mathrm{~s}, 1 \mathrm{H}), 6.74(\mathrm{~s}, 1 \mathrm{H}), 6.11(\mathrm{dd}, J=8.2,0.8 \mathrm{~Hz}, 1 \mathrm{H}), 6.02(\mathrm{~d}, J=8.8 \mathrm{~Hz}, 1 \mathrm{H})$, $3.80(\mathrm{~s}, 3 \mathrm{H}), 3.66(\mathrm{~s}, 3 \mathrm{H}) ;{ }^{13} \mathrm{C}$ NMR (151 MHz, DMSO): $\delta 149.84,149.15,142.98,142.52$, 129.71, 127.76, 127.49, 126.15, 122.37, 120.87, 117.52, 116.95, 116.46, 115.56, 113.59, 113.04, 101.49, 56.05, 55.43; IR (neat, $\mathrm{cm}^{-1}$ ): $v: 3414,1506,1458,1237,747,668$; GC-MS (EI): 431.2, 350.2, 335.1, 278.1, 198.1, 153.1; HRMS (m/z): $[\mathrm{M}]^{+}$calculated for $\mathrm{C}_{20} \mathrm{H}_{16} \mathrm{BrNO}_{3} \mathrm{~S}, 429.0034$; found 429.0028 . 
<smiles>COc1cc(O)c(N2c3ccccc3Sc3ccc(C(C)=O)cc32)cc1OC</smiles>

Compound 3s. Isolated yield: $94 \%$, yellow foamy solid. Prepared following the general procedure using 1-(10H-phenothiazin-2-yl)ethanone ( $96 \mathrm{mg}, 0.4 \mathrm{mmol}$ ), 3,4-dimethoxyphenol (123 mg, 0.8 $\mathrm{mmol})$ and $\mathrm{K}_{2} \mathrm{~S}_{2} \mathrm{O}_{8}(324 \mathrm{mg}, 1.2 \mathrm{mmol})$ in $\mathrm{MeCN}(8.0 \mathrm{ml})$. The reaction mixture was stirred open to air for $48 \mathrm{~h}$. The crude product was purified by flash column chromatography (petroleum: DCM: EA, 8:10:1) to provide the title compound with $147 \mathrm{mg} .{ }^{1} \mathrm{H}$ NMR $\left(400 \mathrm{MHz}, \mathrm{CDCl}_{3}\right): \delta 7.43(\mathrm{dd}$, $J=8.0,1.7 \mathrm{~Hz}, 1 \mathrm{H}), 7.11(\mathrm{~d}, J=7.9 \mathrm{~Hz}, 1 \mathrm{H}), 7.04(\mathrm{dd}, J=7.4,1.7 \mathrm{~Hz}, 1 \mathrm{H}), 6.98-6.86(\mathrm{~m}, 3 \mathrm{H})$, $6.82(\mathrm{~s}, 1 \mathrm{H}), 6.77(\mathrm{~s}, 1 \mathrm{H}), 6.37(\mathrm{dd}, J=8.1,1.3 \mathrm{~Hz}, 1 \mathrm{H}), 5.51(\mathrm{~s}, 1 \mathrm{H}), 3.95(\mathrm{~s}, 3 \mathrm{H}), 3.85(\mathrm{~s}, 3 \mathrm{H})$, 2.39 (s, 3H); ${ }^{13} \mathrm{C}$ NMR $\left(151 \mathrm{MHz}, \mathrm{CDCl}_{3}\right): \delta 196.88,150.74,147.65,144.08,143.61,142.90$, $127.85,126.90,126.70,123.66,123.53,116.06,114.54,113.04,100.97,56.73,56.11,26.44$; IR (neat, $\mathrm{cm}^{-1}$ ): $v: 3413,1678,1509,1465,1236,748$; GC-MS (EI): 393.2, 377.1, 335.1, 318.1, 240.1, 198.1; HRMS (m/z): [M+H] calculated for $\mathrm{C}_{22} \mathrm{H}_{20} \mathrm{NO}_{4} \mathrm{~S}, 394.1113$; found 394.1117.<smiles>COc1cc(O)c(N2c3ccccc3Sc3ccc(C#N)cc32)cc1OC</smiles>

Compound 3t. Isolated yield: $94 \%$, yellow foamy solid. Prepared following the general procedure using $10 \mathrm{H}$-phenothiazine-2-carbonitrile ( $89 \mathrm{mg}, 0.4 \mathrm{mmol}$ ), 3,4-dimethoxyphenol (123 mg, 0.8 $\mathrm{mmol})$ and $\mathrm{K}_{2} \mathrm{~S}_{2} \mathrm{O}_{8}(324 \mathrm{mg}, 1.2 \mathrm{mmol})$ in $\mathrm{MeCN}(8.0 \mathrm{ml})$. The reaction mixture was stirred open to air for $72 \mathrm{~h}$. The crude product was purified by flash column chromatography (petroleum: DCM, $5: 1)$ to provide the title compound with $120 \mathrm{mg}$, with $87 \%$ recovery of 10H-phenothiazine-2-carbonitrile (12 mg). ${ }^{1} \mathrm{H}$ NMR (400 MHz, DMSO): $\delta 9.62(\mathrm{~s}, 1 \mathrm{H}), 7.19$ (dt, $J=17.2,4.7 \mathrm{~Hz}, 2 \mathrm{H}), 6.99(\mathrm{dd}, J=7.5,1.4 \mathrm{~Hz}, 1 \mathrm{H}), 6.96-6.90(\mathrm{~m}, 1 \mathrm{H}), 6.83(\mathrm{td}, J=7.5,1.0 \mathrm{~Hz}$, $1 \mathrm{H}), 6.80(\mathrm{~s}, 1 \mathrm{H}), 6.76(\mathrm{~s}, 1 \mathrm{H}), 6.21(\mathrm{~d}, J=1.4 \mathrm{~Hz}, 1 \mathrm{H}), 6.09(\mathrm{~d}, J=7.5 \mathrm{~Hz}, 1 \mathrm{H}), 3.82(\mathrm{~s}, 3 \mathrm{H})$, $3.68(\mathrm{~s}, 3 \mathrm{H}) ;{ }^{13} \mathrm{C}$ NMR (151 MHz, DMSO): $\delta 150.07,148.98,143.51,143.15,141.88,127.90$, $127.07,126.28,126.04,125.60,122.95,118.78,117.02,116.80,115.85,115.72,113.21,109.45$, 101.59, 56.02, 55.42; IR (neat, $\mathrm{cm}^{-1}$ ): $v: 3426,2224,1507,1463,1235,745$; GC-MS (EI): 376.2, 361.1, 344.1, 301.1, 223.1, 192.1; HRMS $(\mathrm{m} / \mathrm{z})$ : [M] $]^{+}$calculated for $\mathrm{C}_{21} \mathrm{H}_{16} \mathrm{~N}_{2} \mathrm{O}_{3} \mathrm{~S}, 376.0882$; found 376.0882 . 
<smiles>COc1cc(O)c(N2c3ccccc3Sc3ccc(C(F)(F)F)cc32)cc1OC</smiles>

Compound 3u. Isolated yield: $86 \%$, dark viscous oil. Prepared following the general procedure using 2-(trifluoromethyl)-10H-phenothiazine (106 mg, $0.4 \mathrm{mmol}$ ), 3,4-dimethoxyphenol (123 mg, $0.8 \mathrm{mmol})$ and $\mathrm{K}_{2} \mathrm{~S}_{2} \mathrm{O}_{8}(324 \mathrm{mg}, 1.2 \mathrm{mmol})$ in $\mathrm{MeCN}(8.0 \mathrm{ml})$. The reaction mixture was stirred open to air for $72 \mathrm{~h}$. The crude product was purified by flash column chromatography (petroleum: EA, 5:1) to provide the title compound with $122 \mathrm{mg}$, with $85 \%$ recovery of 2-(trifluoromethyl)-10H-phenothiazine (16 mg). ${ }^{1} \mathrm{H}$ NMR (400 MHz, DMSO): $\delta 9.66$ (s, 1H), 7.19 $(\mathrm{d}, J=7.9 \mathrm{~Hz}, 1 \mathrm{H}), 7.10(\mathrm{dd}, J=8.0,1.1 \mathrm{~Hz}, 1 \mathrm{H}), 7.00(\mathrm{dd}, J=7.5,1.5 \mathrm{~Hz}, 1 \mathrm{H}), 6.97-6.90(\mathrm{~m}$, $1 \mathrm{H}), 6.84(\mathrm{dd}, J=7.5,1.2 \mathrm{~Hz}, 1 \mathrm{H}), 6.81(\mathrm{~d}, J=2.9 \mathrm{~Hz}, 1 \mathrm{H}), 6.78(\mathrm{~s}, 1 \mathrm{H}), 6.31(\mathrm{~d}, J=1.4 \mathrm{~Hz}, 1 \mathrm{H})$, $6.11(\mathrm{dd}, J=8.2,1.0 \mathrm{~Hz}, 1 \mathrm{H}), 3.83(\mathrm{~s}, 3 \mathrm{H}), 3.67$ (s, 3H); ${ }^{13} \mathrm{C}$ NMR (151 MHz, DMSO): $\delta 150.05$, 149.23, 143.61, 143.07, 142.26, 127.92, 127.72, 126.85, 126.25, 124.84, 124.19 (d, $J=1.5 \mathrm{~Hz}$ ), 123.04, 122.85, 118.87-118.00 (m), 117.41, 116.00, 115.85, 113.36, 110.85 (q, $J=4.1 \mathrm{~Hz}), 101.33$, 56.03, 55.41, 54.86; ${ }^{19} \mathrm{~F}$ NMR (376 MHz, DMSO): $\delta$-61.60; IR (neat, $\mathrm{cm}^{-1}$ ): $v: 3408,1509,1468$, 1120, 955, 748; GC-MS (EI): 419.2, 266.2, 235.1, 209.6, 95.0, 69.0; HRMS (m/z): [M] $]^{+}$calculated for $\mathrm{C}_{21} \mathrm{H}_{16} \mathrm{~F}_{3} \mathrm{NO}_{3} \mathrm{~S}$, 419.0803; found 419.0803 .<smiles>COc1ccc2c(c1)Nc1c(cccc1N1c3ccccc3Sc3ccc(OC)cc31)S2</smiles>

Compound 3v. Isolated yield: 79\%, dark viscous oil. Prepared following the general procedure using 2-methoxy-10H-phenothiazine ( $91 \mathrm{mg}, 0.4 \mathrm{mmol}$ ), 3,4-dimethoxyphenol (123 mg, $0.8 \mathrm{mmol}$ ) and $\mathrm{K}_{2} \mathrm{~S}_{2} \mathrm{O}_{8}$ (324 mg, $\left.1.2 \mathrm{mmol}\right)$ in $\mathrm{MeCN}(8.0 \mathrm{ml})$. The reaction mixture was stirred open to air for $1 \mathrm{~h}$. The crude product was purified by flash column chromatography (petroleum: EA, 4:1) to provide the title compound with $48 \mathrm{mg}$, with $66 \%$ recovery of 2-methoxy-10H-phenothiazine (31 mg). ${ }^{1} \mathrm{H}$ NMR (400 MHz, DMSO): $\delta 8.94$ (s, 1H), 7.03 (t, $\left.J=7.6 \mathrm{~Hz}, 1 \mathrm{H}\right), 6.99-6.87$ (m, 5H), $6.83-6.77(\mathrm{~m}, 2 \mathrm{H}), 6.75(\mathrm{~d}, J=7.9 \mathrm{~Hz}, 1 \mathrm{H}), 6.67(\mathrm{~s}, 1 \mathrm{H}), 6.45(\mathrm{~d}, J=8.4 \mathrm{~Hz}, 1 \mathrm{H}), 6.12(\mathrm{~d}, J=$ $8.2 \mathrm{~Hz}, 1 \mathrm{H}), 5.66$ (s, 1H), 3.70 (s, 3H), 3.57 (s, 3H); ${ }^{13} \mathrm{C}$ NMR (151 MHz, DMSO): $\delta 158.98$, $156.64,144.11,144.07,142.54,141.40,128.37,127.60,127.15,126.73,126.28,126.14,122.28$, 122.26, 121.29, 118.93, 116.55, 115.34, 114.69, 109.36, 108.12, 106.26, 102.97, 99.66, 55.51, 55.03; IR (neat, $\left.\mathrm{cm}^{-1}\right): v: 3439,2925,1463,1258,1207,745$; HRMS $(m / z):[\mathrm{M}+\mathrm{Na}]^{+}$calculated for $\mathrm{C}_{26} \mathrm{H}_{20} \mathrm{~N}_{2} \mathrm{NaO}_{2} \mathrm{~S}_{2}, 479.0864$; found 479.0860 . 


\section{$\square$.Gram scale reaction}

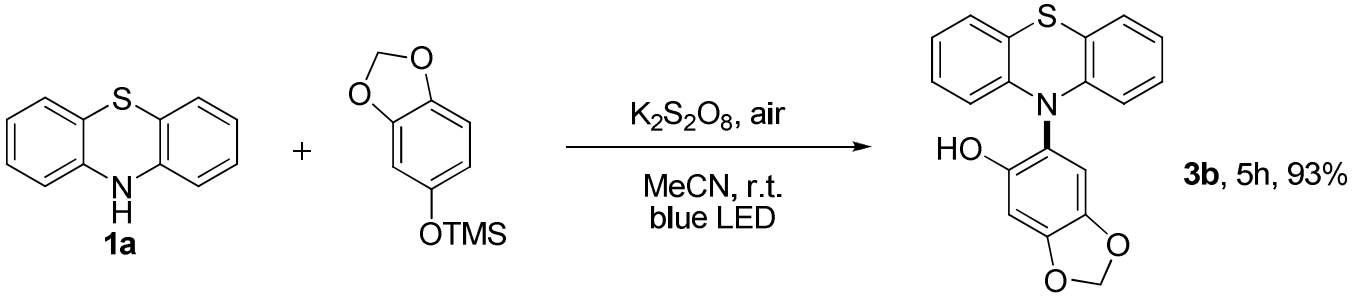

Phenothiazine 1a (0.50 g, $2.5 \mathrm{mmol})$, TMS-protected sesamol (1.05 g, $5.0 \mathrm{mmol})$ and $\mathrm{K}_{2} \mathrm{~S}_{2} \mathrm{O}_{8}$ ( $2.02 \mathrm{~g}, 7.5 \mathrm{mmol})$ were combined in dried flask. $30 \mathrm{ml} \mathrm{MeCN}$ was added via syringe. The flask was capped with a rubber that pierced through by a needle, and then exposed to $8 \mathrm{~W}$ blue LED strips for irradiation in air. After reaction for $5 \mathrm{~h}$, the mixture was diluted with DCM and filtered through an inch of silica gel. The crude product was purified by flash column chromatography (petroleum: EA, 5:1) to provide $\mathbf{3 b}$ as white solid in $93 \%$ yield $(0.77 \mathrm{~g})$.

\section{Control Experiments}<smiles>c1ccc2c(c1)Nc1ccccc1S2</smiles>

$1 \mathrm{a}$

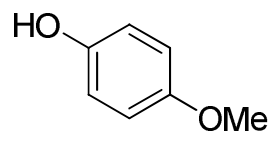

$2 \mathrm{a}$

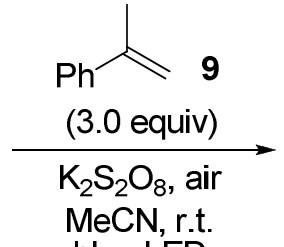

blue LED

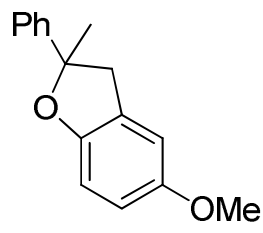

10

Phenothiazine 1a (40 mg, $0.2 \mathrm{mmol})$, phenol 2a ( $50 \mathrm{mg}, 0.4 \mathrm{mmol})$, prop-1-en-2-ylbenzene 9 (70 $\mathrm{mg}, 0.6 \mathrm{mmol})$ and $\mathrm{K}_{2} \mathrm{~S}_{2} \mathrm{O}_{8}(162 \mathrm{mg}, 0.6 \mathrm{mmol})$ were combined in dried flask. $4 \mathrm{ml} \mathrm{MeCN}$ was added via syringe. The flask was capped with a rubber that pierced through by a needle, and then exposed to $8 \mathrm{~W}$ blue LED strips for irradiation in air. After reaction for $72 \mathrm{~h}$, the mixture was diluted with DCM and filtered through an inch of silica gel. Then the filtrate was used for GC-MS analysis.

GC-MS (10) caculated for, 240.1150; found 240.2 as Fig. S1 showed. ${ }^{[1]}$ 


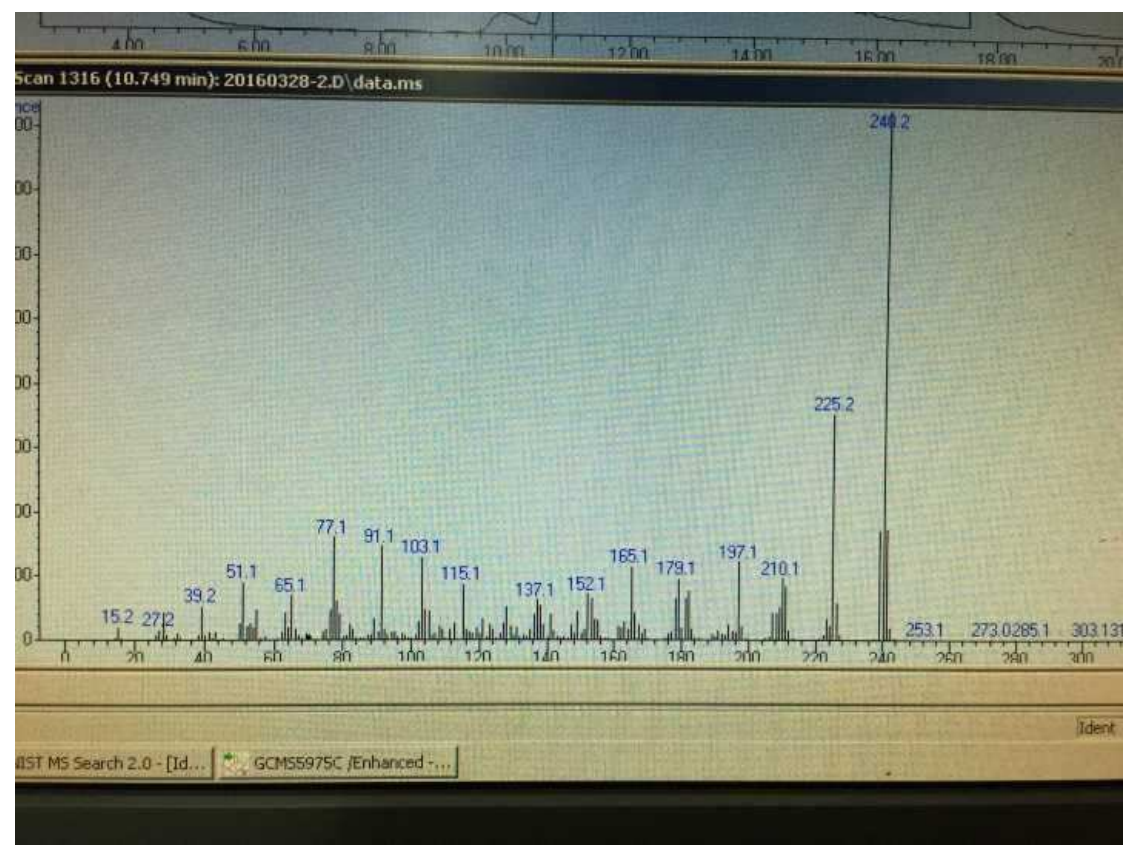

Fig S1. Detected GC-MS data for 10.<smiles>c1ccc2c(c1)Nc1ccccc1S2</smiles><smiles>COc1ccc(O)cc1</smiles>

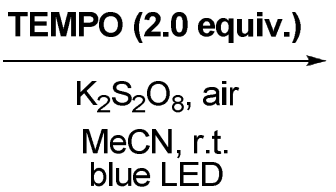<smiles>COc1ccc(O)c(N2c3ccccc3Sc3ccccc32)c1</smiles>

$3 a, 1 \mathrm{~h}, 62 \%$

Phenothiazine 1a (40 mg, $0.2 \mathrm{mmol})$, phenol 2a (50 mg, $0.4 \mathrm{mmol})$, TEMPO (62 mg, $0.4 \mathrm{mmol})$ and $\mathrm{K}_{2} \mathrm{~S}_{2} \mathrm{O}_{8}$ (162 mg, $0.6 \mathrm{mmol}$ ) were combined in dried flask. $4 \mathrm{ml} \mathrm{MeCN}$ was added via syringe. The flask was capped with a rubber that pierced through by a needle, and then exposed to $8 \mathrm{~W}$ blue LED strips for irradiation in air. After reaction for 1h, the mixture was diluted with DCM and filtered through an inch of silica gel. The crude product was purified by flash column chromatography (petroleum: EA, 5:1) to provide 3a as white solid in $62 \%$ yield (39 mg).<smiles>COc1ccc2c(c1)Nc1ccccc1S2</smiles>

$1 v$<smiles>COc1ccc(O)cc1OC</smiles>
$2 \mathrm{~g}$

\section{TEMPO (2.0 equiv.)}

$\mathrm{K}_{2} \mathrm{~S}_{2} \mathrm{O}_{8}$, air $\mathrm{MeCN}$, r.t. blue LED<smiles>COc1ccc2c(c1)N(c1cc(OC)c(OC)cc1O)c1ccccc1S2</smiles>

$3 v^{\prime}, 1 \mathrm{~h}, 58 \%$ cross-coupling product 
Phenothiazine $1 \mathbf{v}$ (46 mg, $0.2 \mathrm{mmol}$ ), phenol 2g (61 mg, $0.4 \mathrm{mmol}$ ), TEMPO (62 mg, $0.4 \mathrm{mmol})$ and $\mathrm{K}_{2} \mathrm{~S}_{2} \mathrm{O}_{8}$ (162 mg, $0.6 \mathrm{mmol}$ ) were combined in dried flask. $4 \mathrm{ml} \mathrm{MeCN}$ was added via syringe. The flask was capped with a rubber that pierced through by a needle, and then exposed to $8 \mathrm{~W}$ blue LED strips for irradiation in air. After reaction for $1 \mathrm{~h}$, the mixture was diluted with DCM and filtered through an inch of silica gel. The crude product was purified by flash column chromatography (petroleum: EA, 4:1) to provide 3v' in 58\% yield (44 mg).

Compound 3v', dark viscous liquid. ${ }^{1} \mathrm{H}$ NMR (400 MHz, $\mathrm{CDCl}_{3}$ ): $\delta 7.08(\mathrm{~d}, J=8.9 \mathrm{~Hz}, 1 \mathrm{H}), 6.98$ $(\mathrm{d}, J=8.4 \mathrm{~Hz}, 1 \mathrm{H}), 6.97-6.85(\mathrm{~m}, 2 \mathrm{H}), 6.83(\mathrm{~s}, 1 \mathrm{H}), 6.75(\mathrm{~s}, 1 \mathrm{H}), 6.48(\mathrm{~d}, J=44.1 \mathrm{~Hz}, 2 \mathrm{H}), 6.04$ $(\mathrm{d}, J=2.2 \mathrm{~Hz}, 1 \mathrm{H}), 5.61(\mathrm{~s}, 1 \mathrm{H}), 3.94(\mathrm{~s}, 3 \mathrm{H}), 3.84(\mathrm{~s}, 3 \mathrm{H}), 3.63(\mathrm{~s}, 3 \mathrm{H}) ;{ }^{13} \mathrm{C} \mathrm{NMR}(151 \mathrm{MHz}$, DMSO): $\delta 158.99,149.70,149.22,144.20,142.86,142.64,127.02,126.54,126.02,122.04$, $118.78,116.91,115.52,113.75,109.29,105.93,103.40,101.43,56.06,55.44,55.00$; IR (neat, $\left.\mathrm{cm}^{-1}\right): v:$ 3394, 1573, 1462, 1261, 1206, 745; GC-MS (EI): 381.2, 349.1, 333.1, 317.1, 228.1, 185.1; HRMS $(\mathrm{m} / z)$ : $[\mathrm{M}]^{+}$calculated for $\mathrm{C}_{21} \mathrm{H}_{19} \mathrm{NO}_{4} \mathrm{~S}, 381.1035$; found 381.1039.
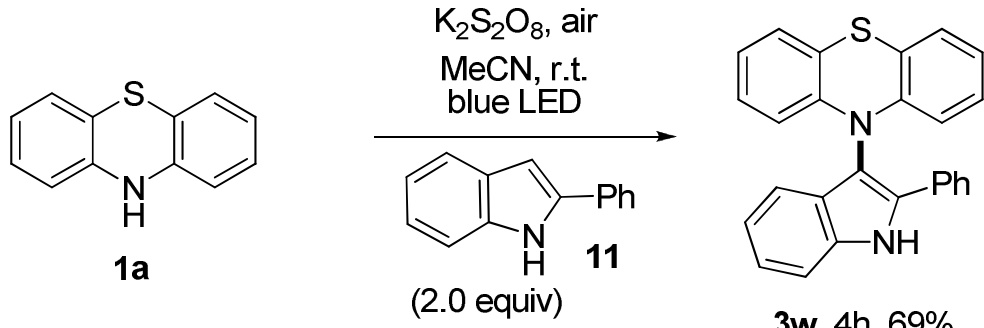

3w, 4h, 69\%

Phenothiazine 1a (40 mg, $0.2 \mathrm{mmol})$, 2-phenyl- $1 H$-indole 11 (77 mg, $0.4 \mathrm{mmol})$ and $\mathrm{K}_{2} \mathrm{~S}_{2} \mathrm{O}_{8}(162$ $\mathrm{mg}, 0.6 \mathrm{mmol}$ ) were combined in dried flask. $4 \mathrm{ml} \mathrm{MeCN}$ was added via syringe. The flask was capped with a rubber that pierced through by a needle, and then exposed to $8 \mathrm{~W}$ blue LED strips for irradiation in air. After reaction for $4 \mathrm{~h}$, the mixture was diluted with DCM and filtered through an inch of silica gel. The crude product was purified by flash column chromatography (petroleum: DCM, 4:1) to provide $3 \mathrm{w}$ in $69 \%$ yield $(53 \mathrm{mg})$.

Compound 3w, yellow solid. ${ }^{1} \mathrm{H}$ NMR (400 MHz, DMSO): $\delta 11.93$ (s, 1H), 7.94 (d, J=7.6 Hz, $2 \mathrm{H}), 7.58(\mathrm{~d}, J=8.1 \mathrm{~Hz}, 1 \mathrm{H}), 7.43(\mathrm{t}, J=7.6 \mathrm{~Hz}, 2 \mathrm{H}), 7.31(\mathrm{t}, J=7.2 \mathrm{~Hz}, 1 \mathrm{H}), 7.25(\mathrm{t}, J=7.7 \mathrm{~Hz}$, 2H), $7.07(\mathrm{dd}, J=14.5,7.6 \mathrm{~Hz}, 3 \mathrm{H}), 6.89-6.78(\mathrm{~m}, 4 \mathrm{H}), 6.29(\mathrm{~d}, J=8.2 \mathrm{~Hz}, 2 \mathrm{H}) ;{ }^{13} \mathrm{C} \mathrm{NMR}(151$ MHz, DMSO): $\delta 143.43,135.10,132.98,130.49,128.88,128.15,127.47,126.60,126.17,125.71$, $122.78,122.72,120.26,119.61,117.99,115.69,112.62,112.44$; IR (neat, $\mathrm{cm}^{-1}$ ): v: 3440, 1586, 1459, 1297, 1250, 743; GC-MS (EI): 390.2, 358.2, 286.1, 254.1, 193.1, 178.2; HRMS (m/z): [M] calculated for $\mathrm{C}_{26} \mathrm{H}_{18} \mathrm{NO}_{2} \mathrm{~S}, 390.1191$; found 390.1189 .

Apart from the TMS-protected sesamol, Ac- and Bz- protected sesamols were also examined in standard conditions, but failed to give corresponding CDC-amination products. Therefore we conducted Cyclic Voltammetry $(\mathrm{CV})$ tests $^{[3]}$ for evaluation of their redox potentials. Cyclic Voltammograms were showed as Fig. S2. 

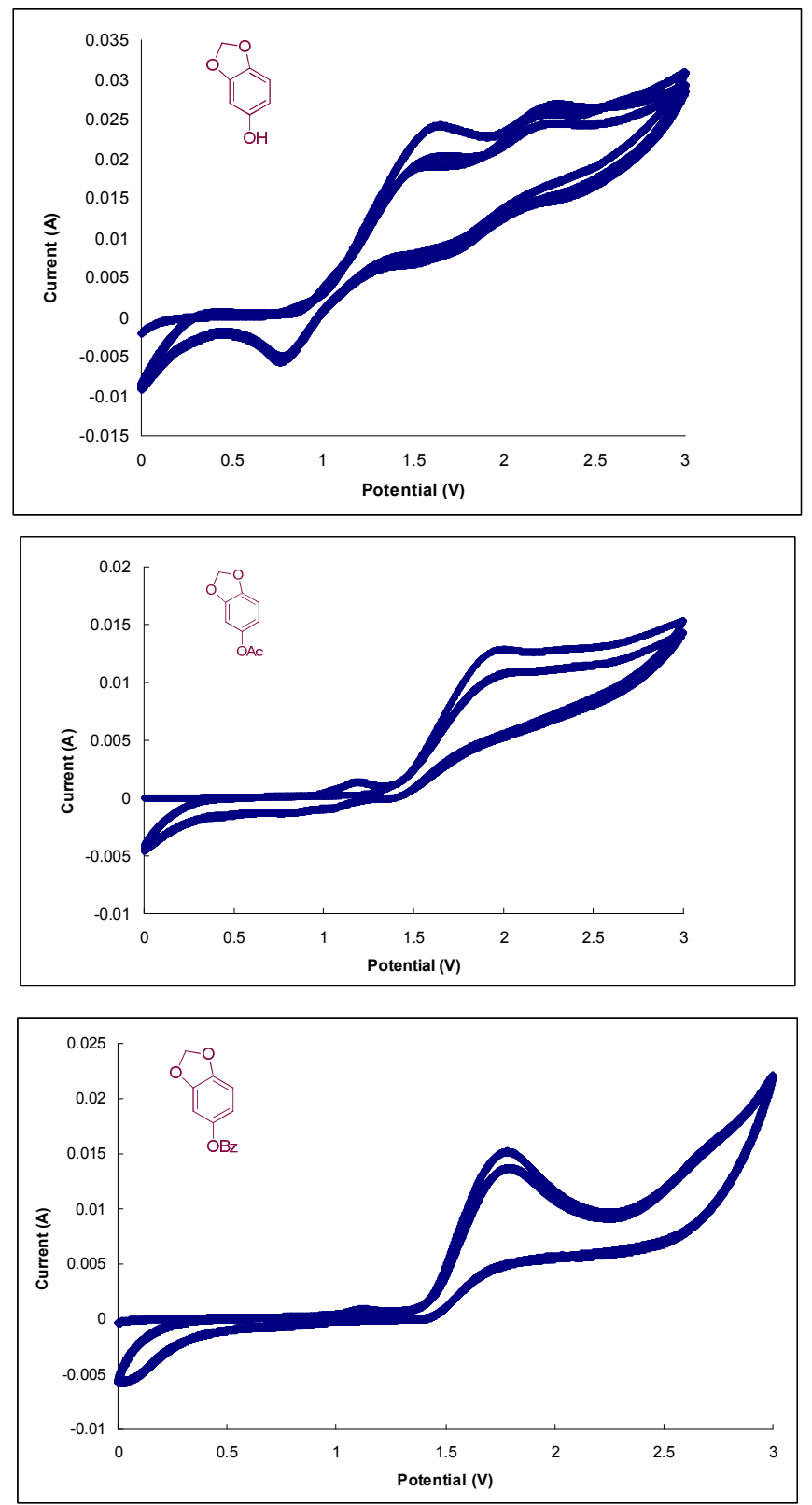

Fig S2. Cyclic Voltammograms for sesamol and Ac-/Bz-protected sesamol.

\section{Reference}

[1] Y. T. Zhao, B. B. Huang, C. Yang, B. Li, W. J. Xia, Synthesis, 2015, 47, 2731.

[2] M. -L. Louillat-Habermeyer, R. W. Jin, F. W. Patureau, Angew. Chem. Int. Ed. 2015, 54, 4102; Angew. Chem. 2015, 127, 4175.

[3] D. S. Hamilton, D. A. Nicewicz, J. Am. Chem. Soc. 2012, 134, 18577. 


\section{NMR Spectra}
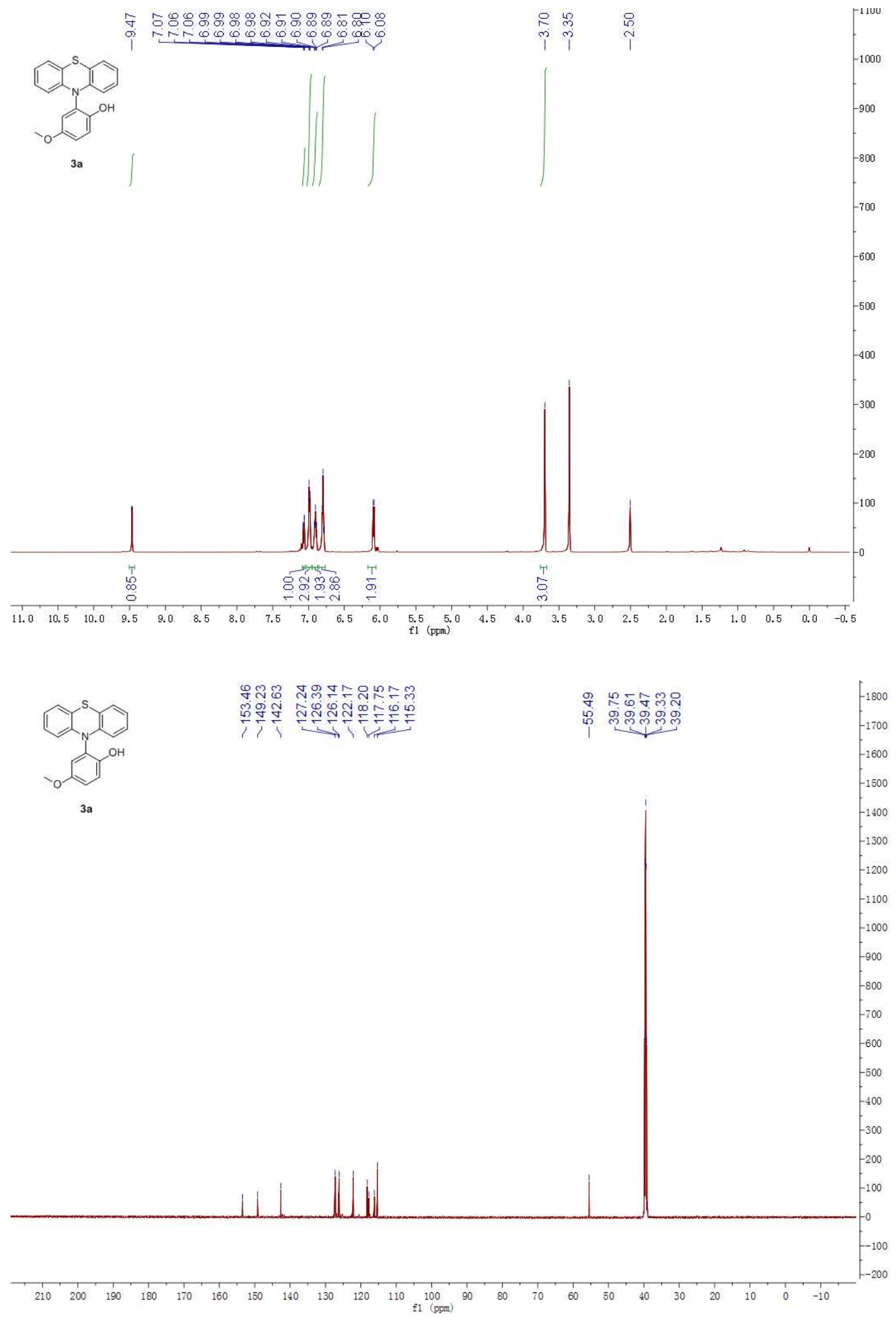


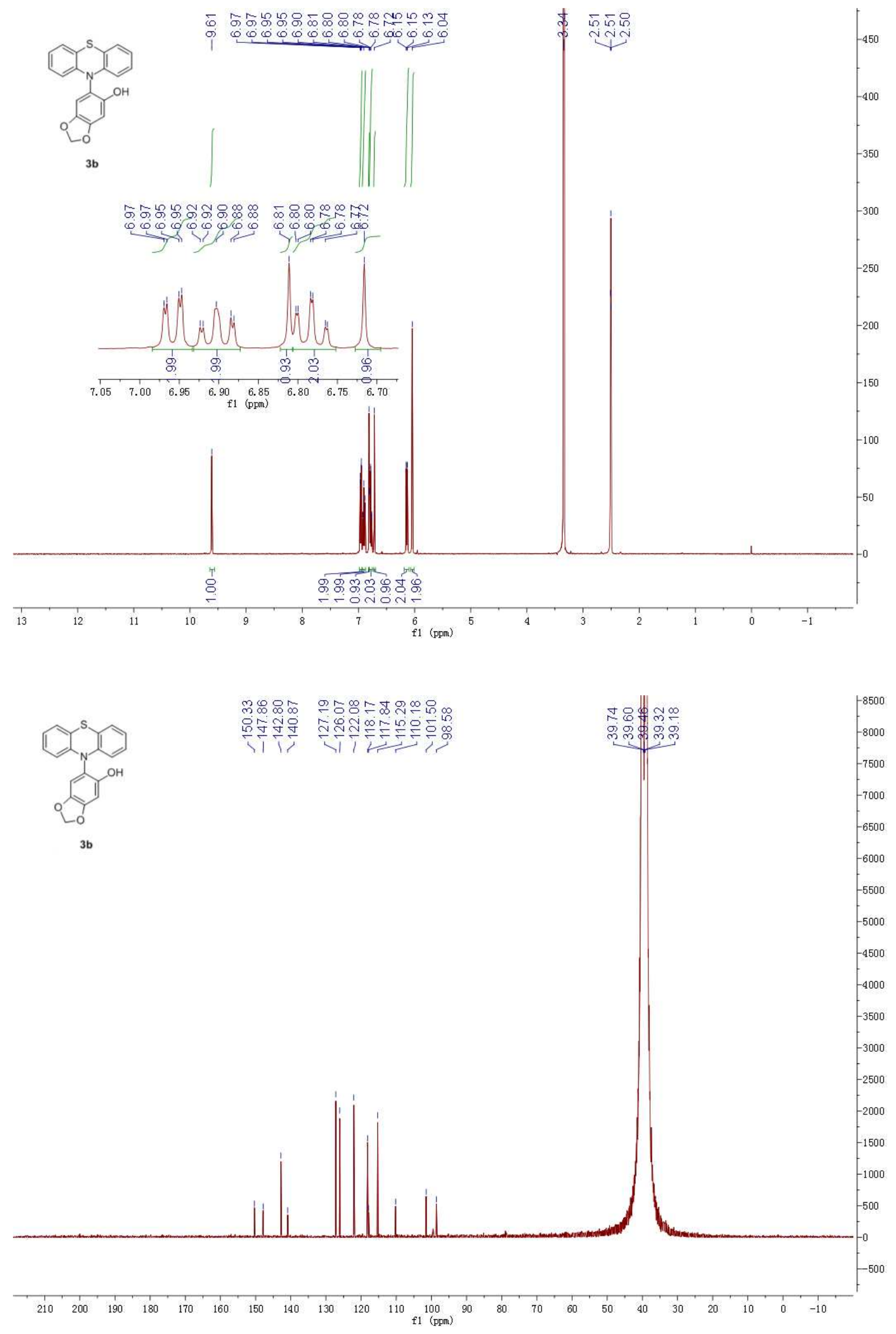




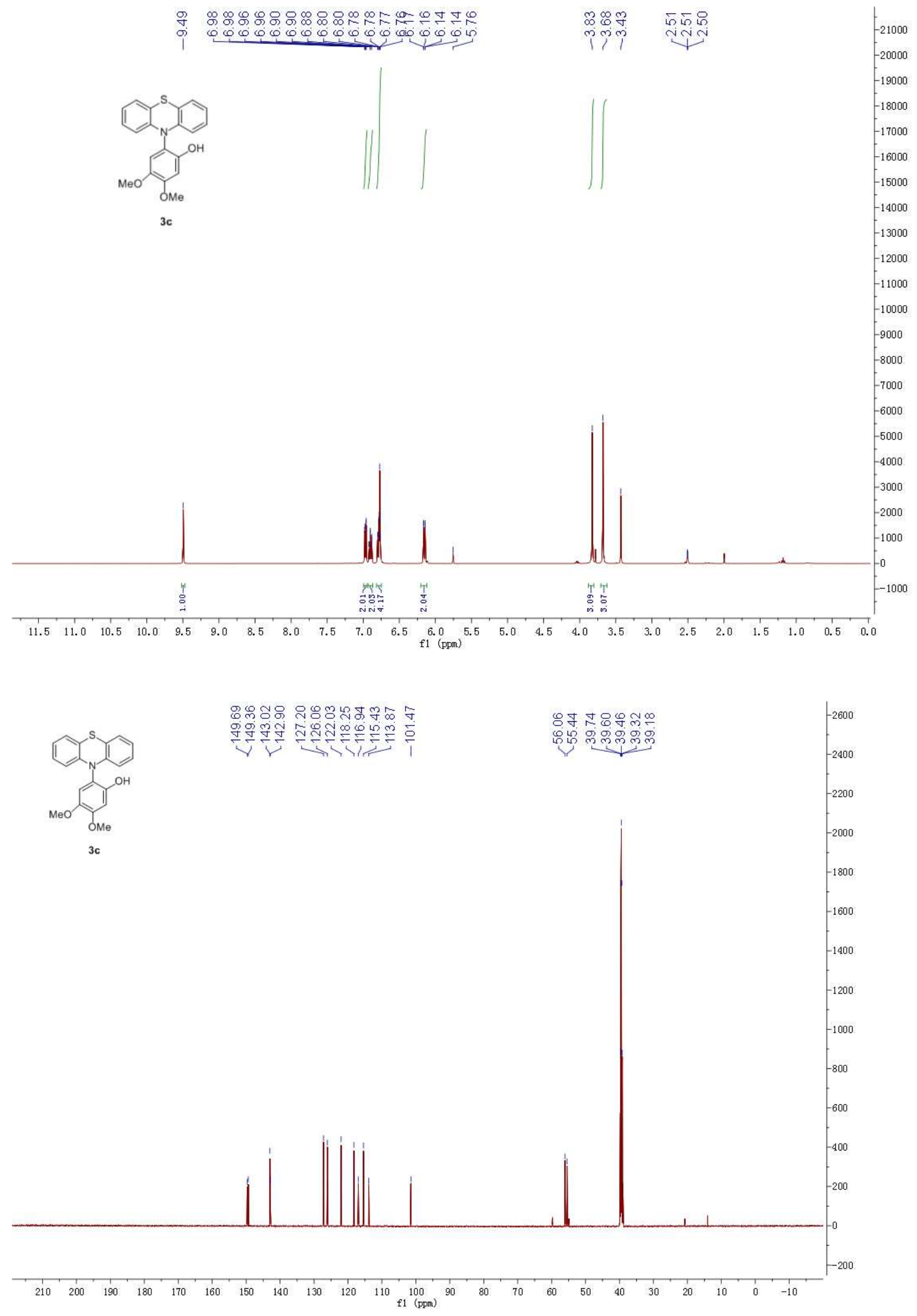



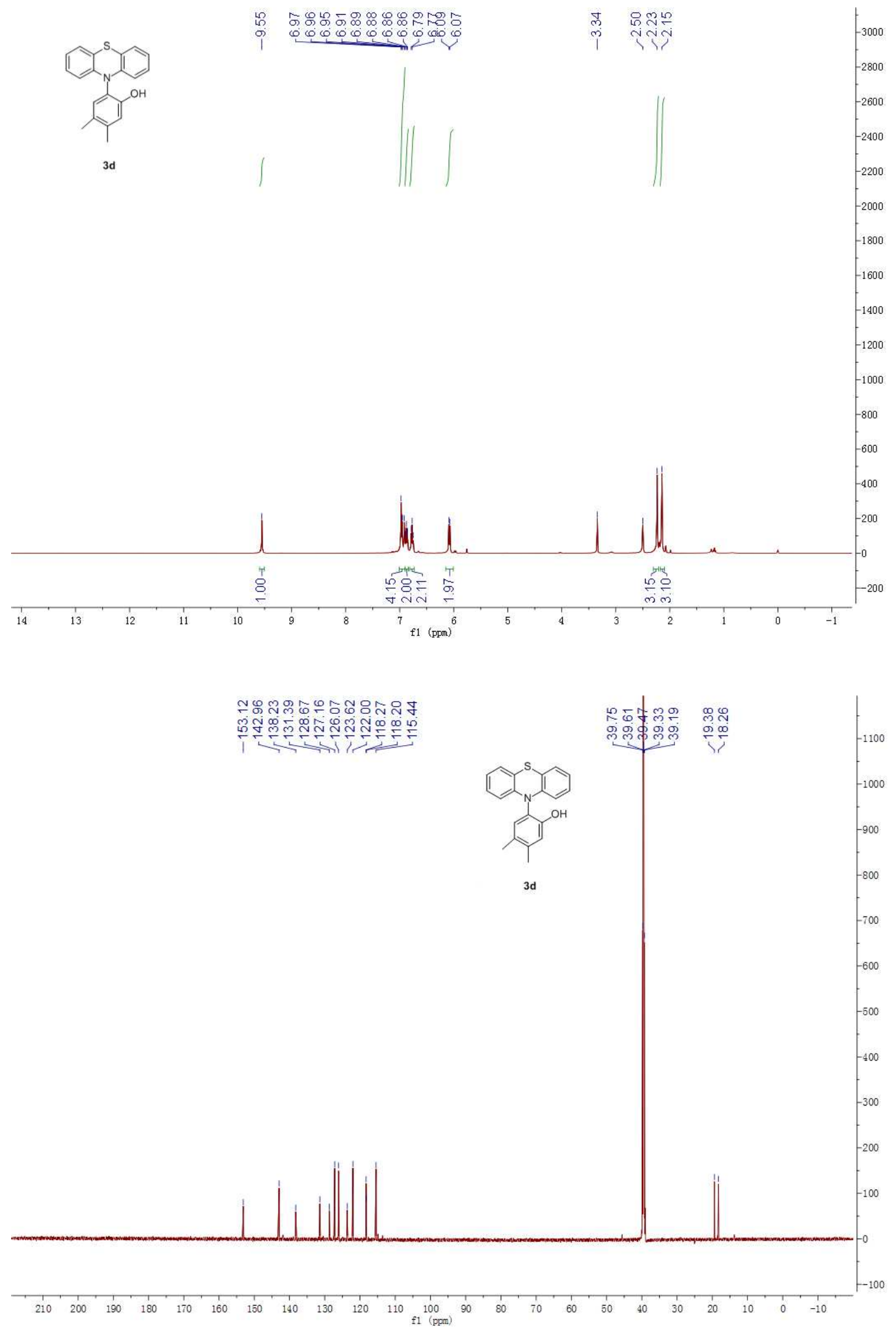

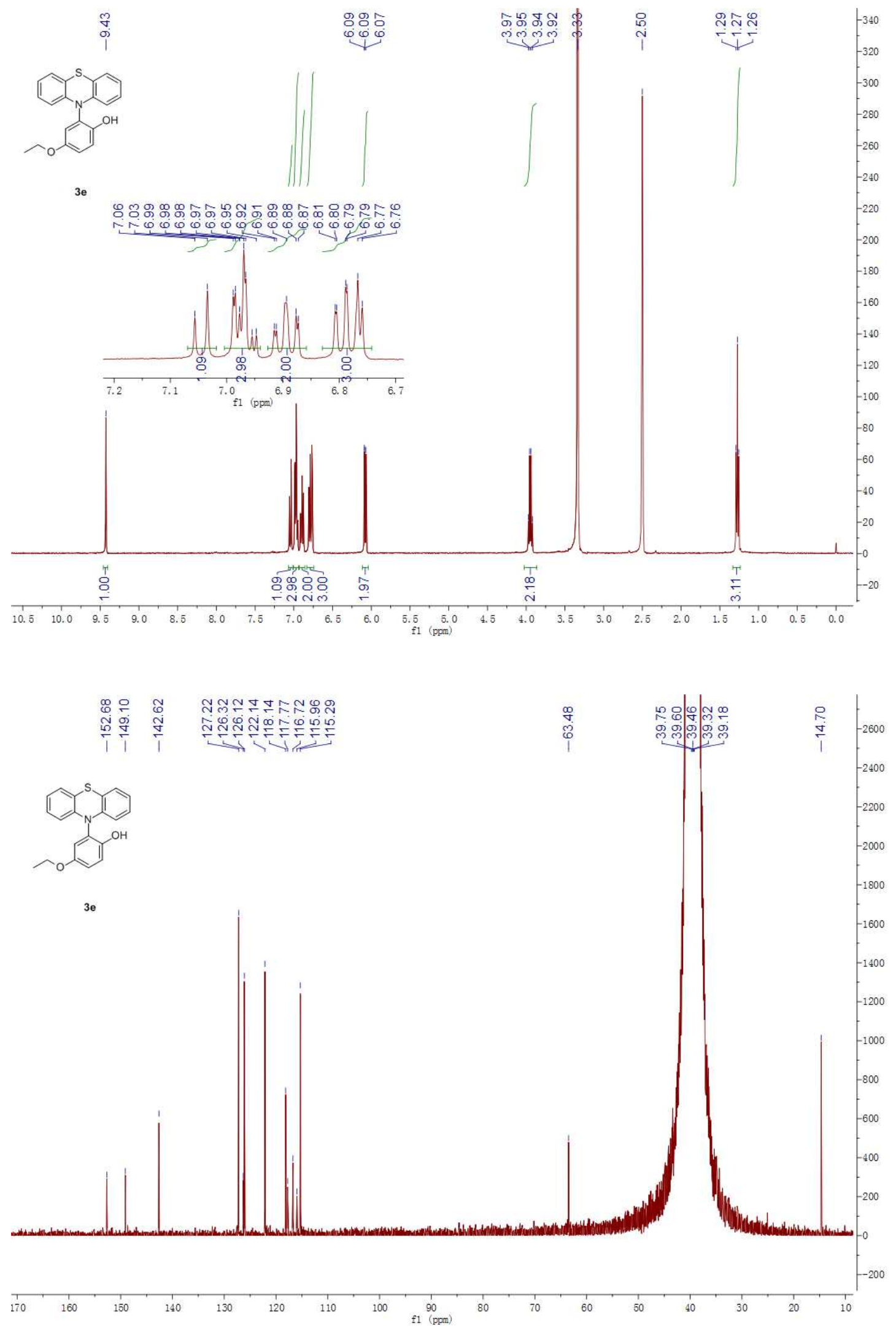

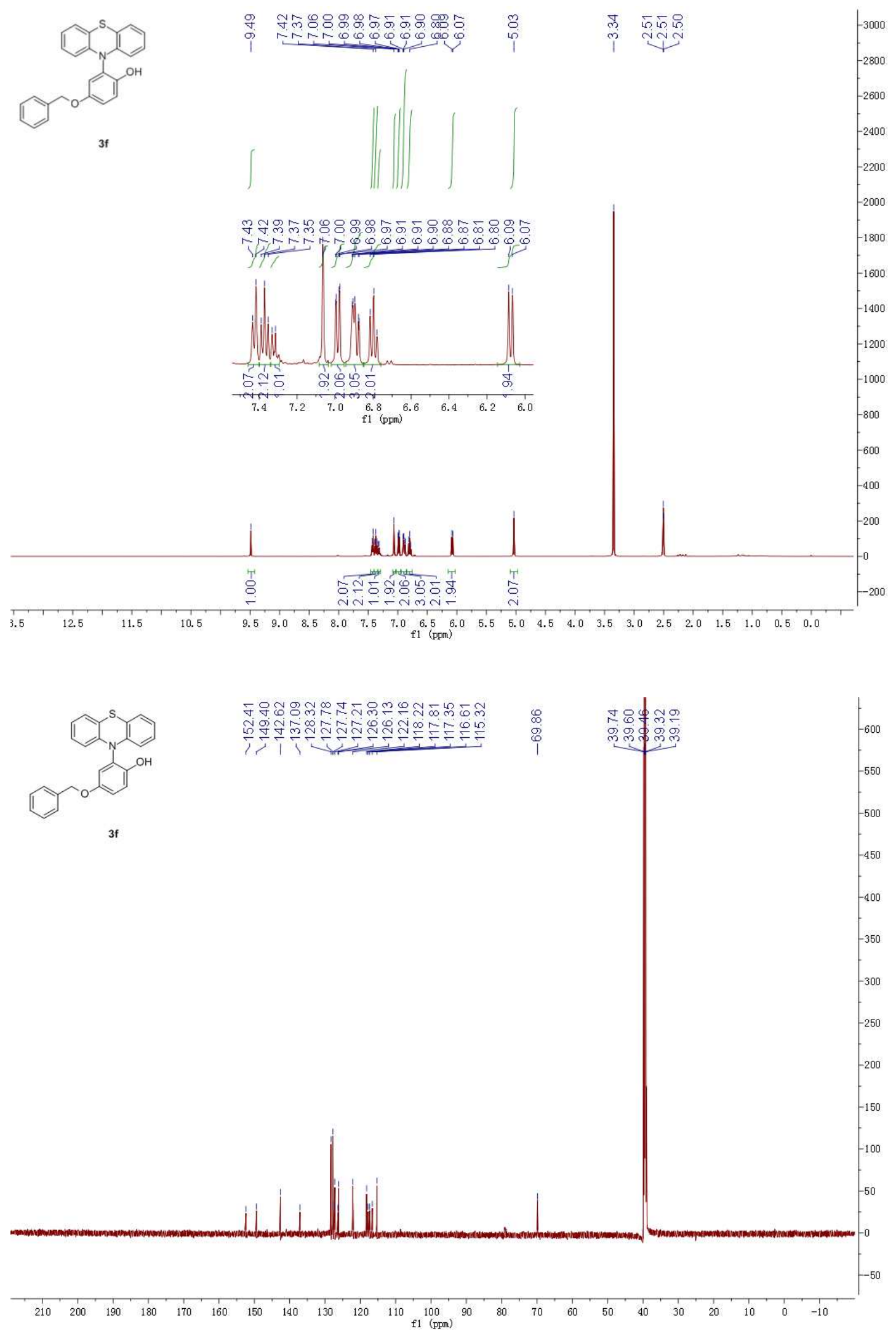


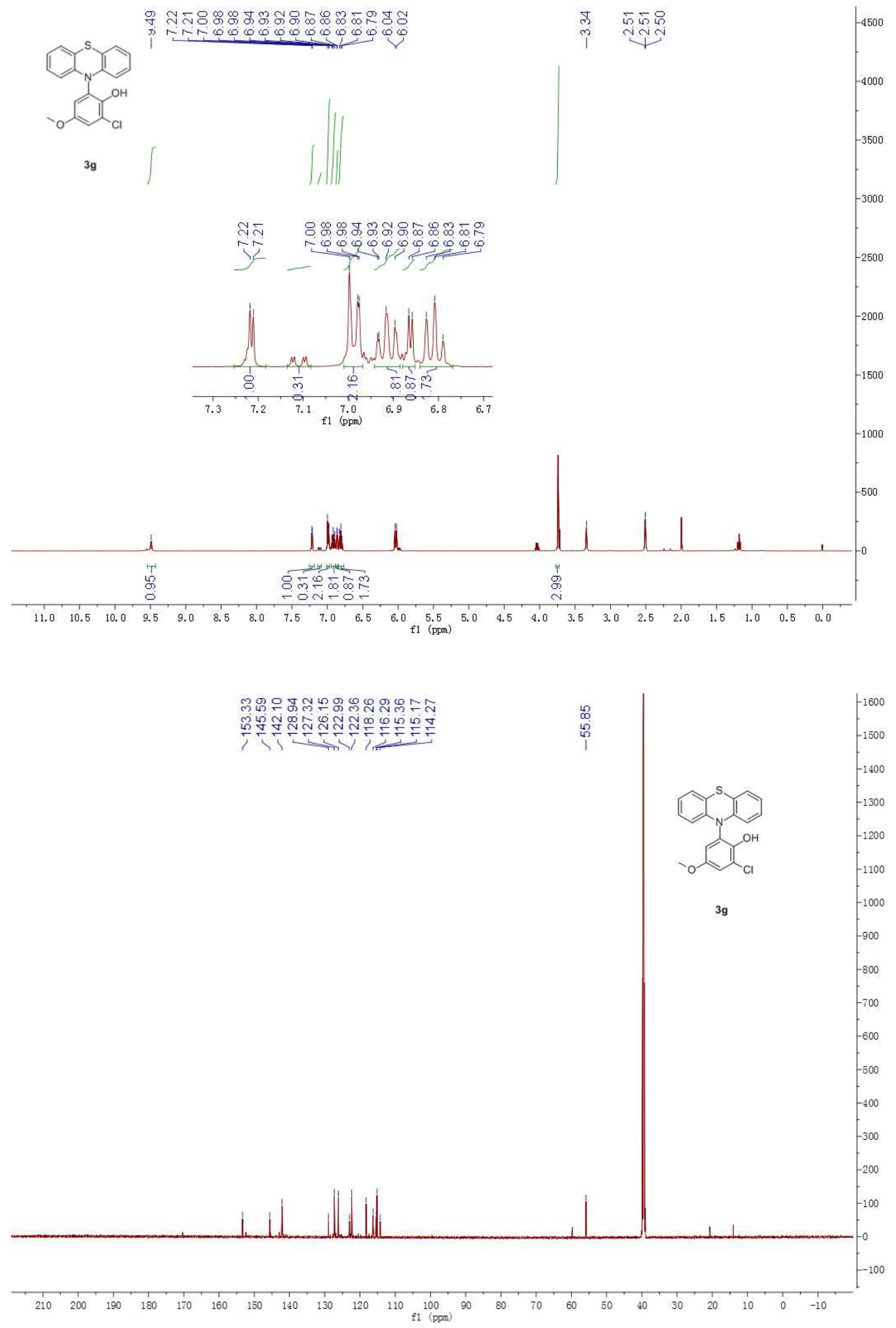



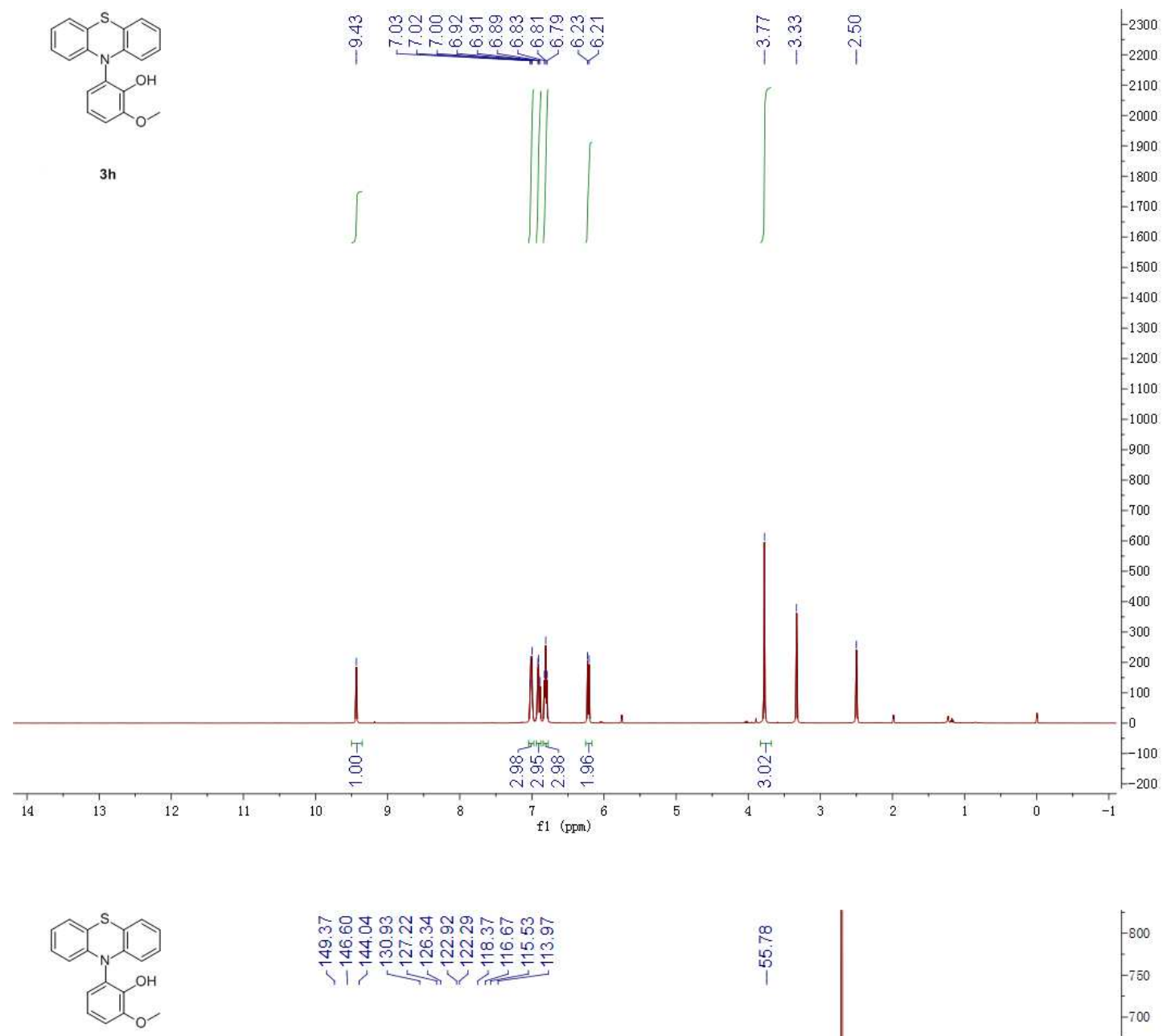

$3 \mathrm{~h}$

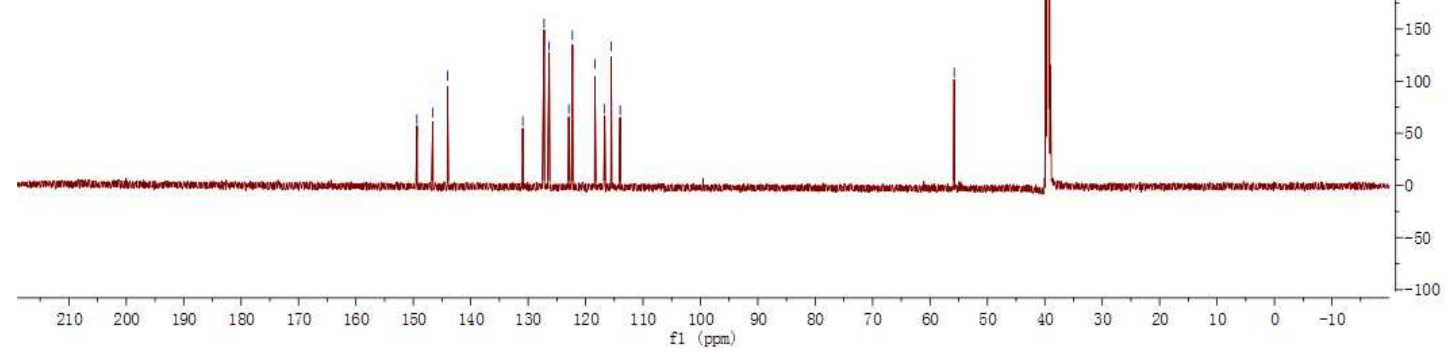




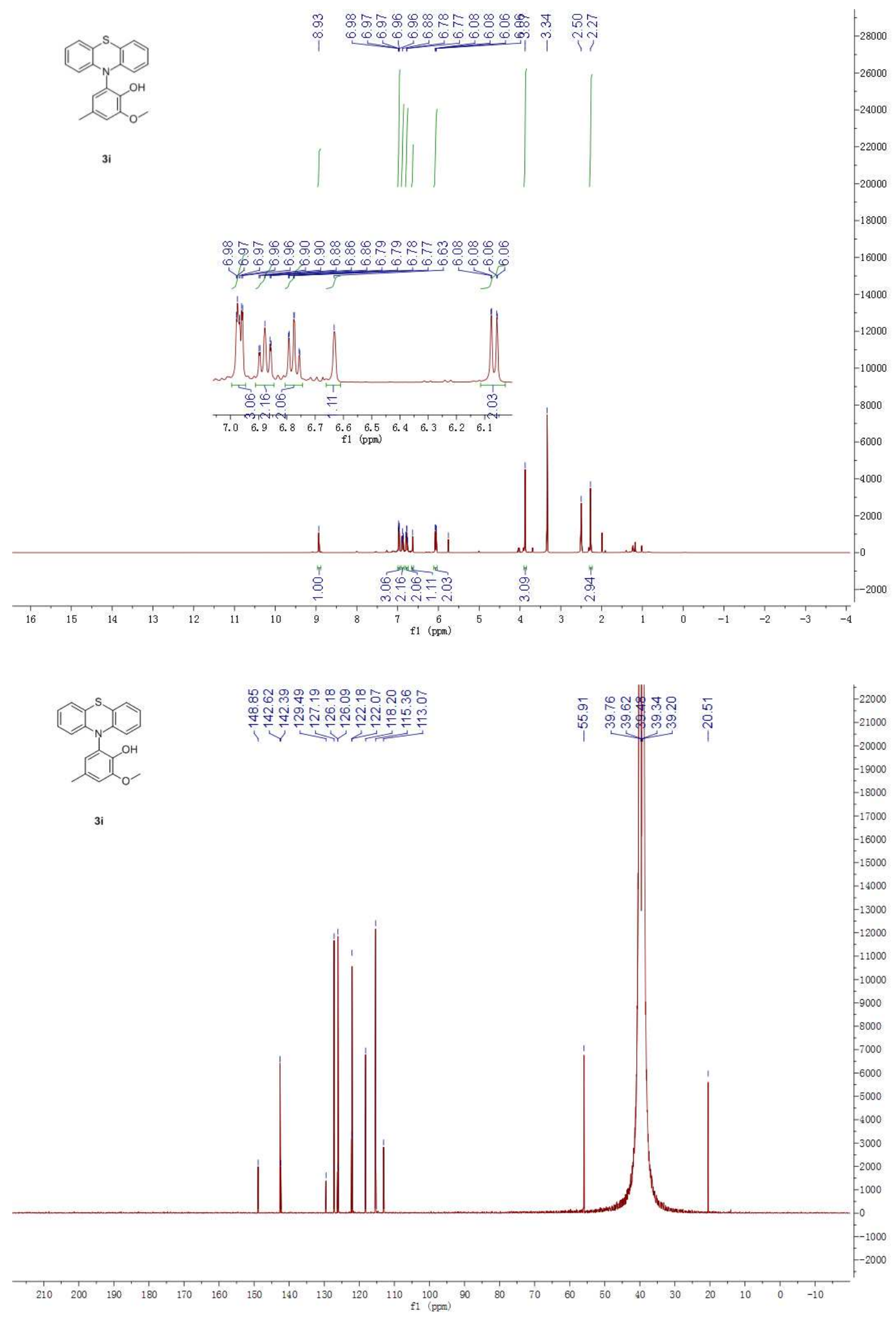



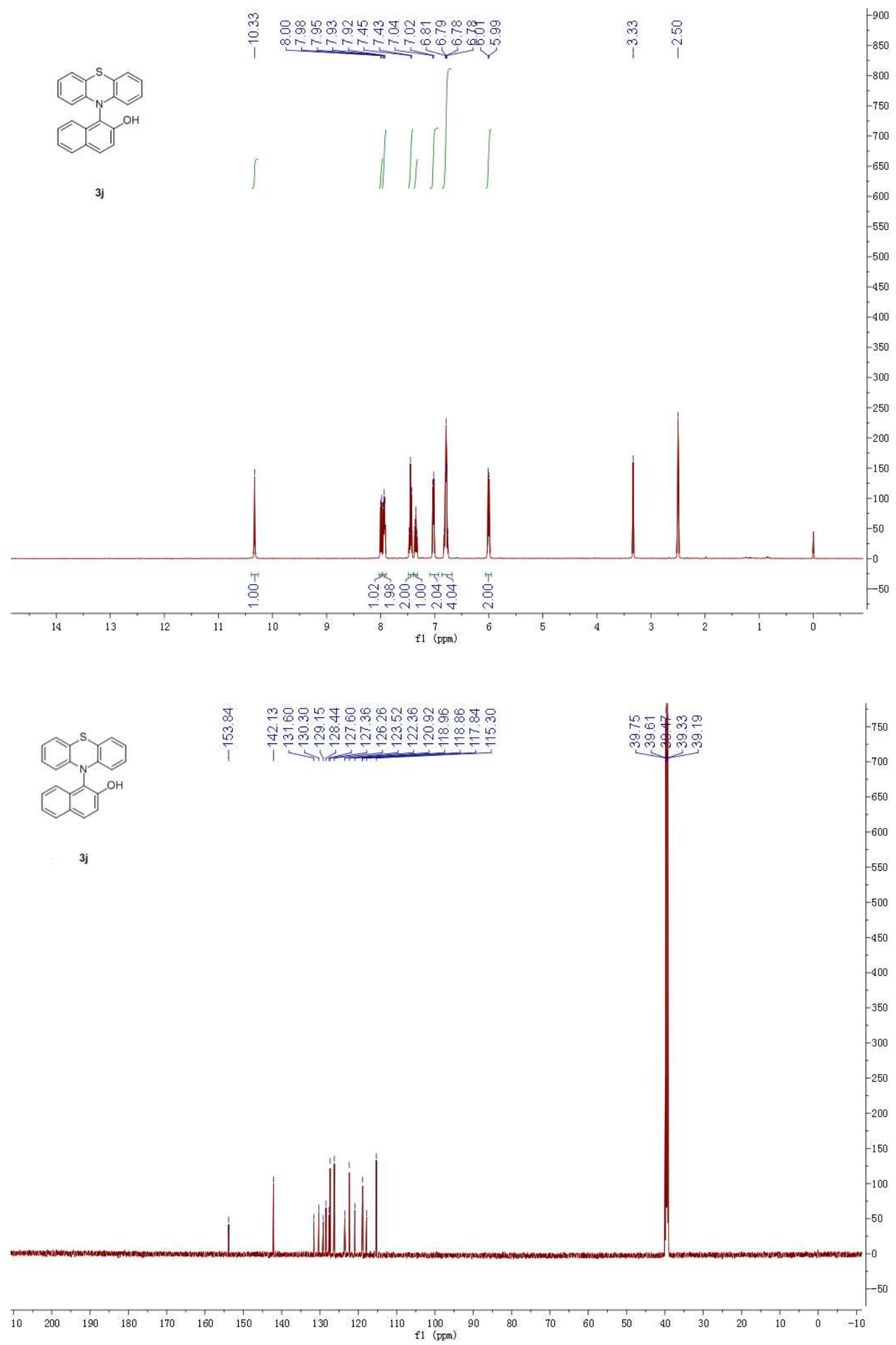

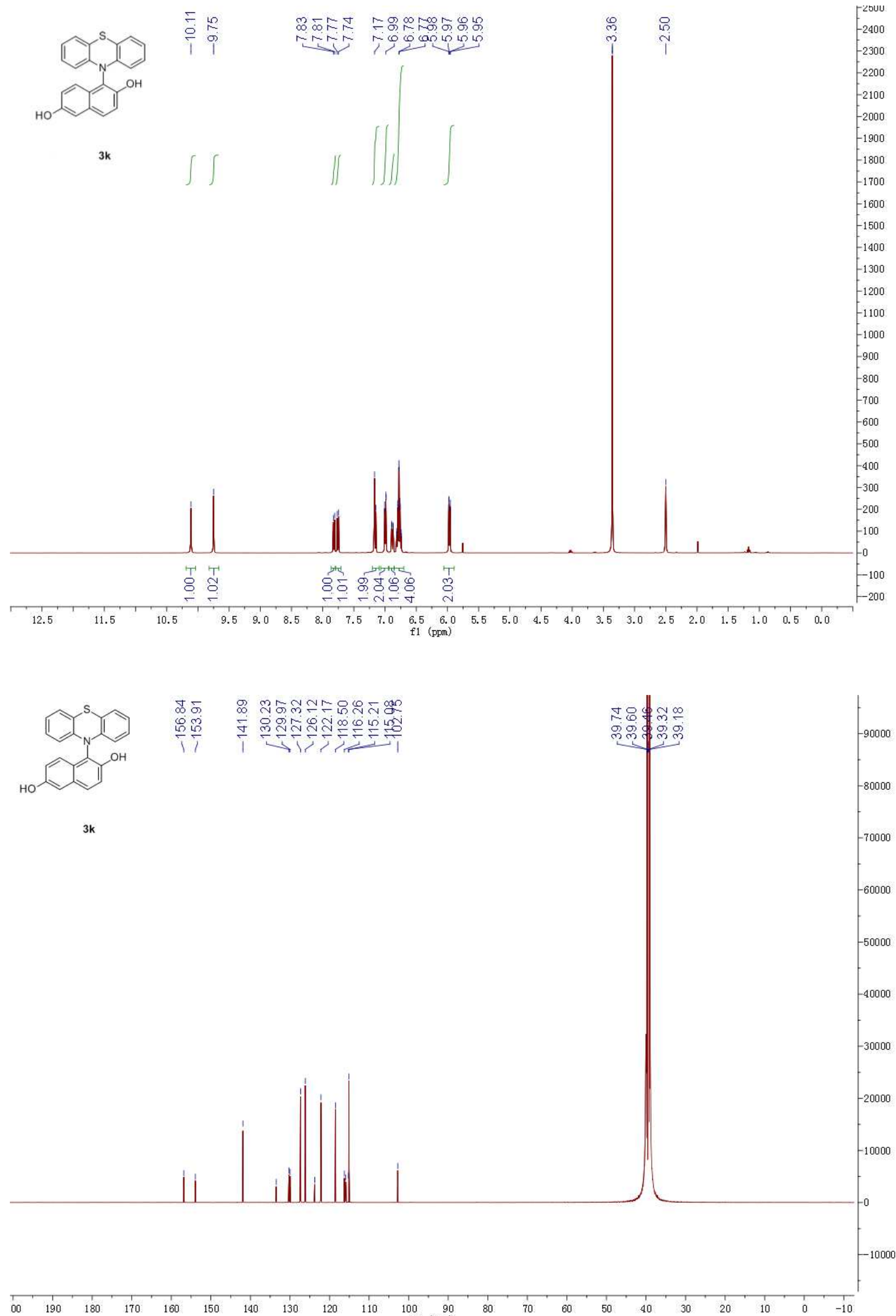

$\begin{array}{llllllllllll}00 & 190 & 180 & 170 & 160 & 150 & 140 & 130 & 120 & 110 & 100 & 90 \\ \mathrm{f} 1 & (\mathrm{ppgm})\end{array}$ 

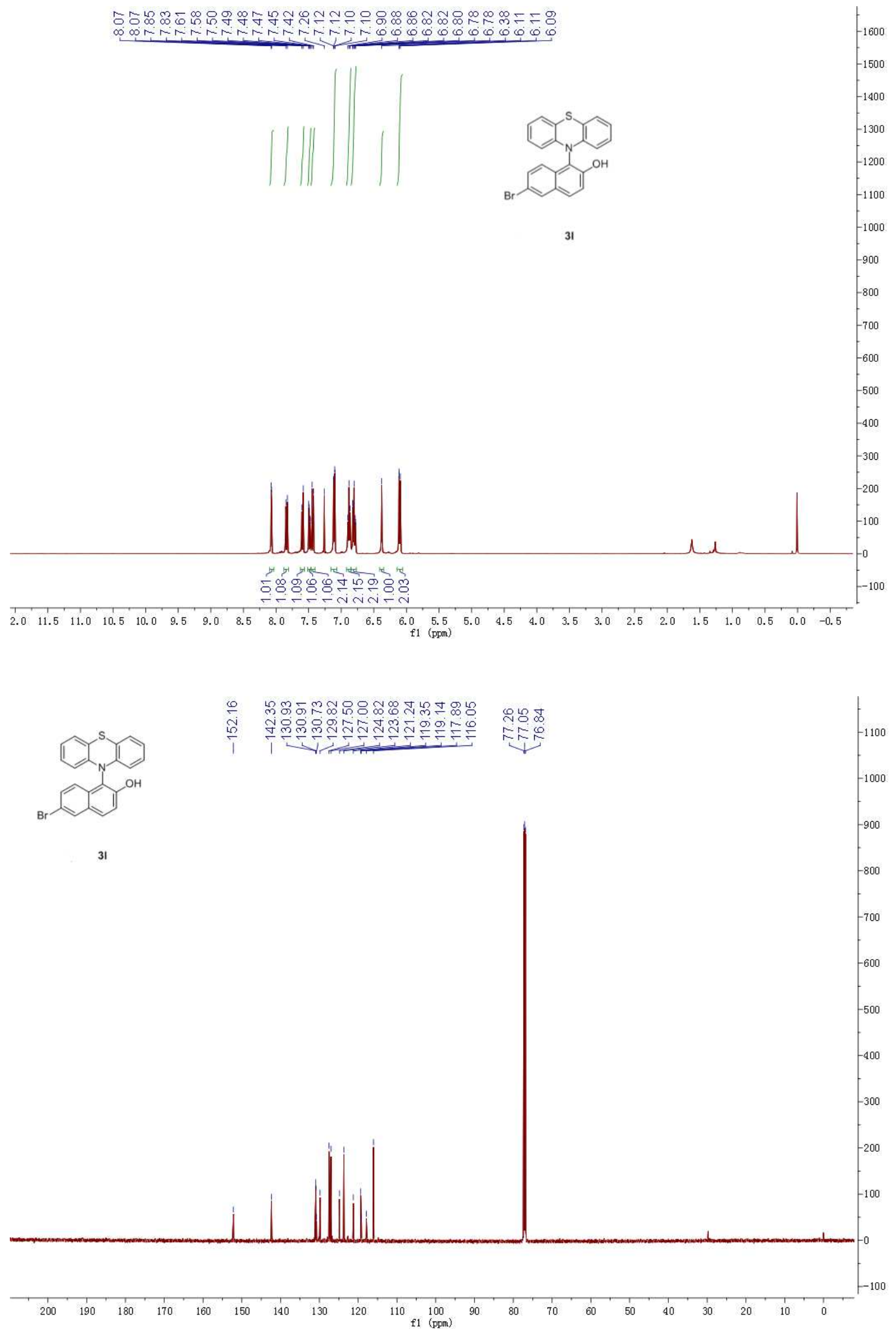


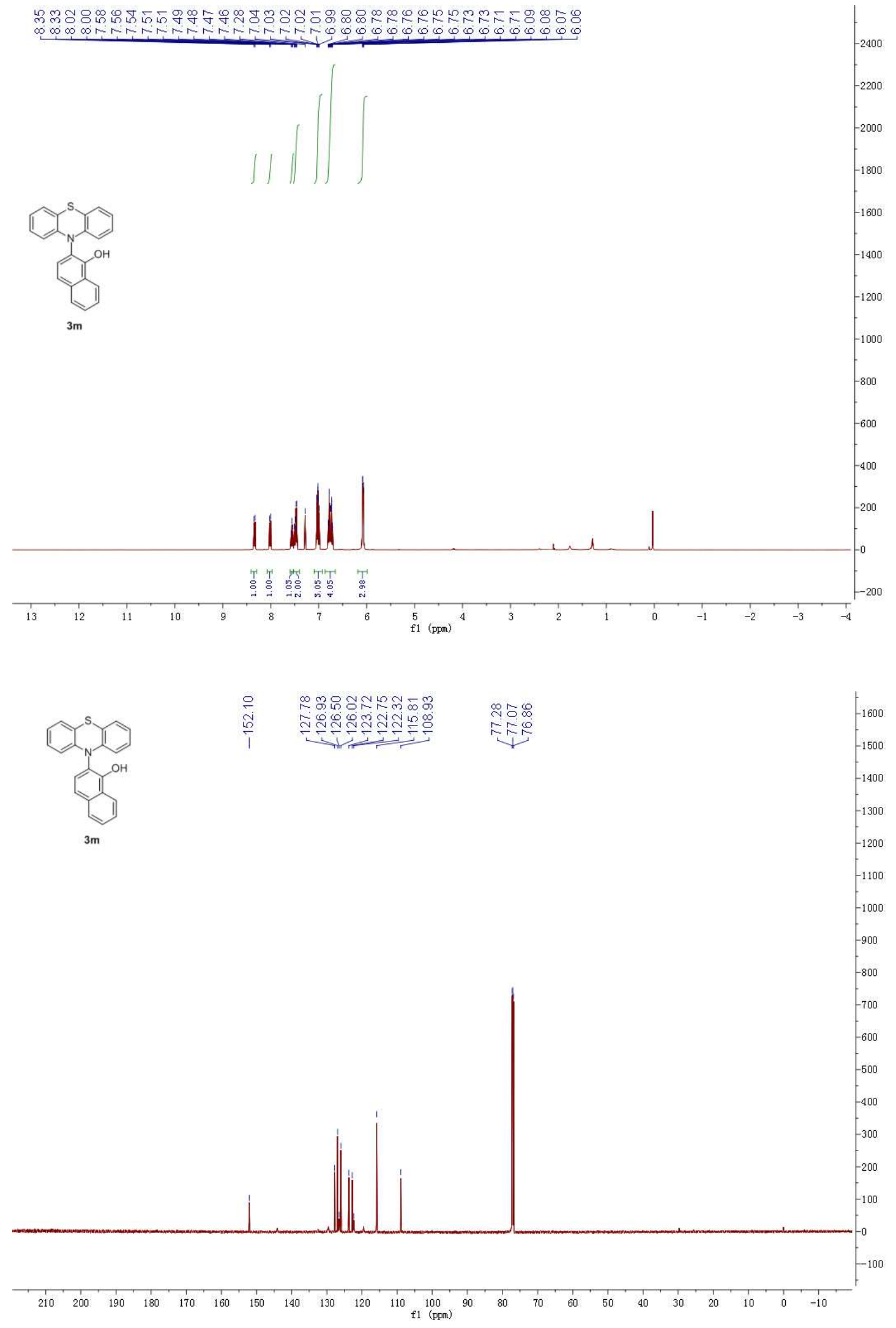



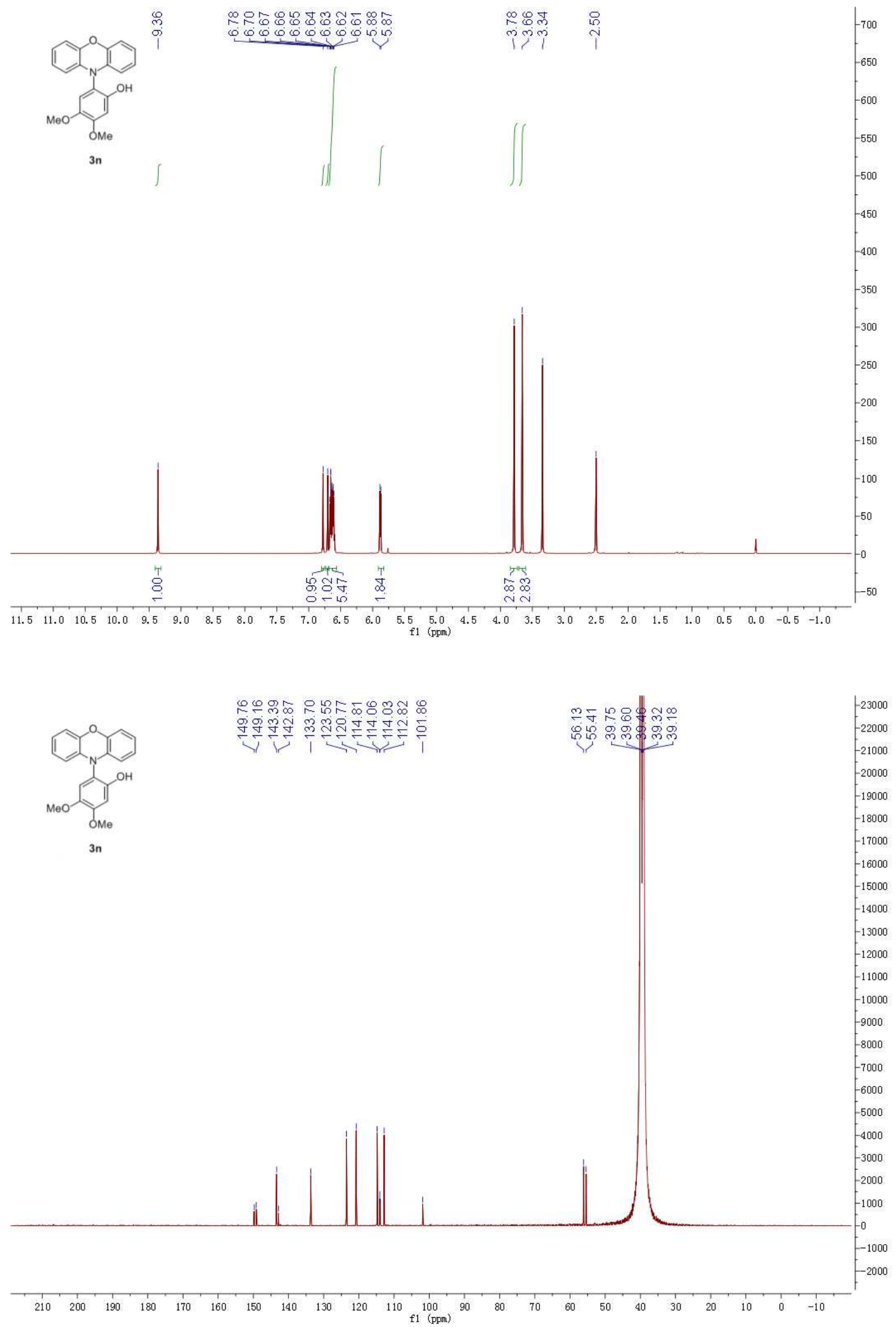


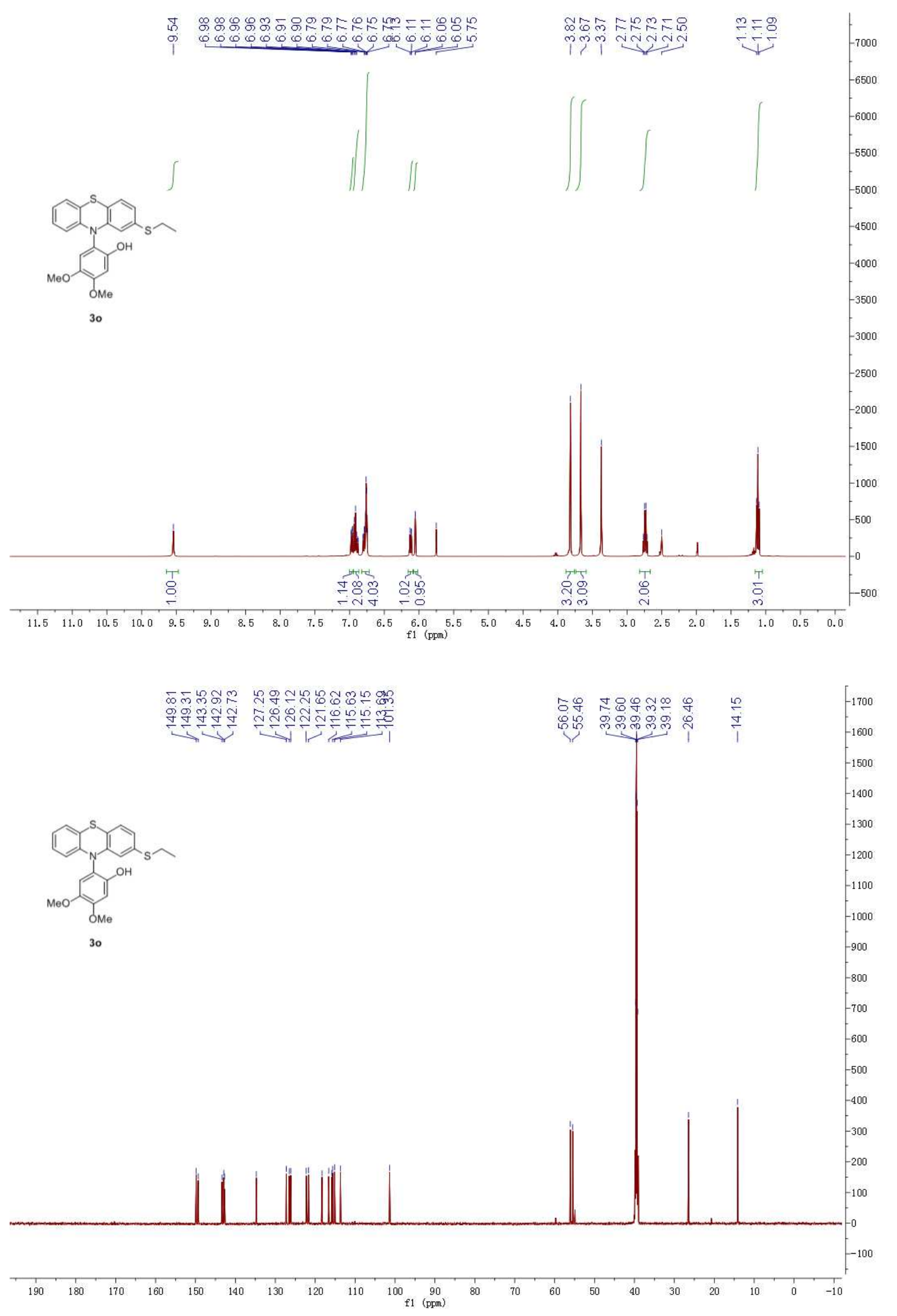



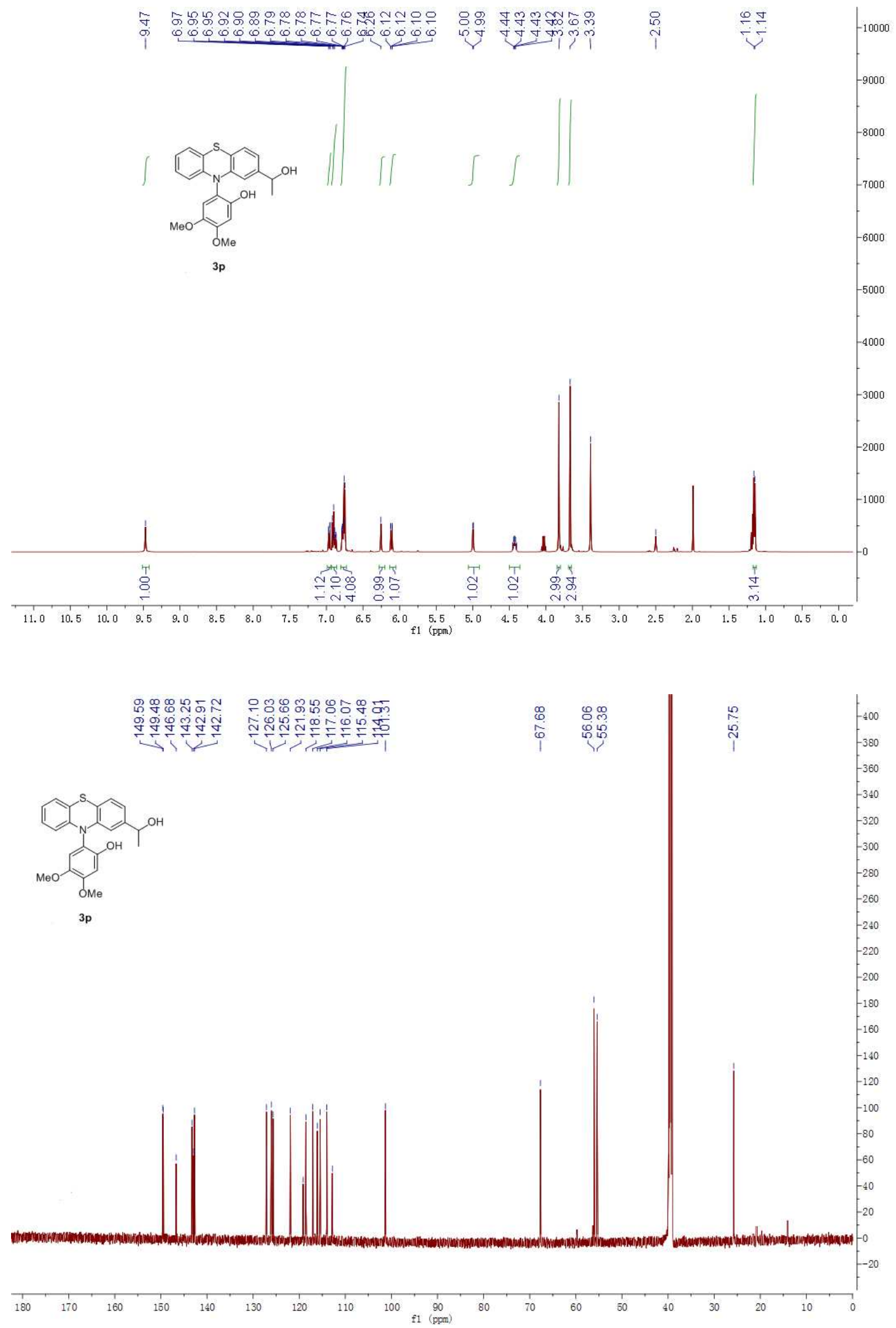


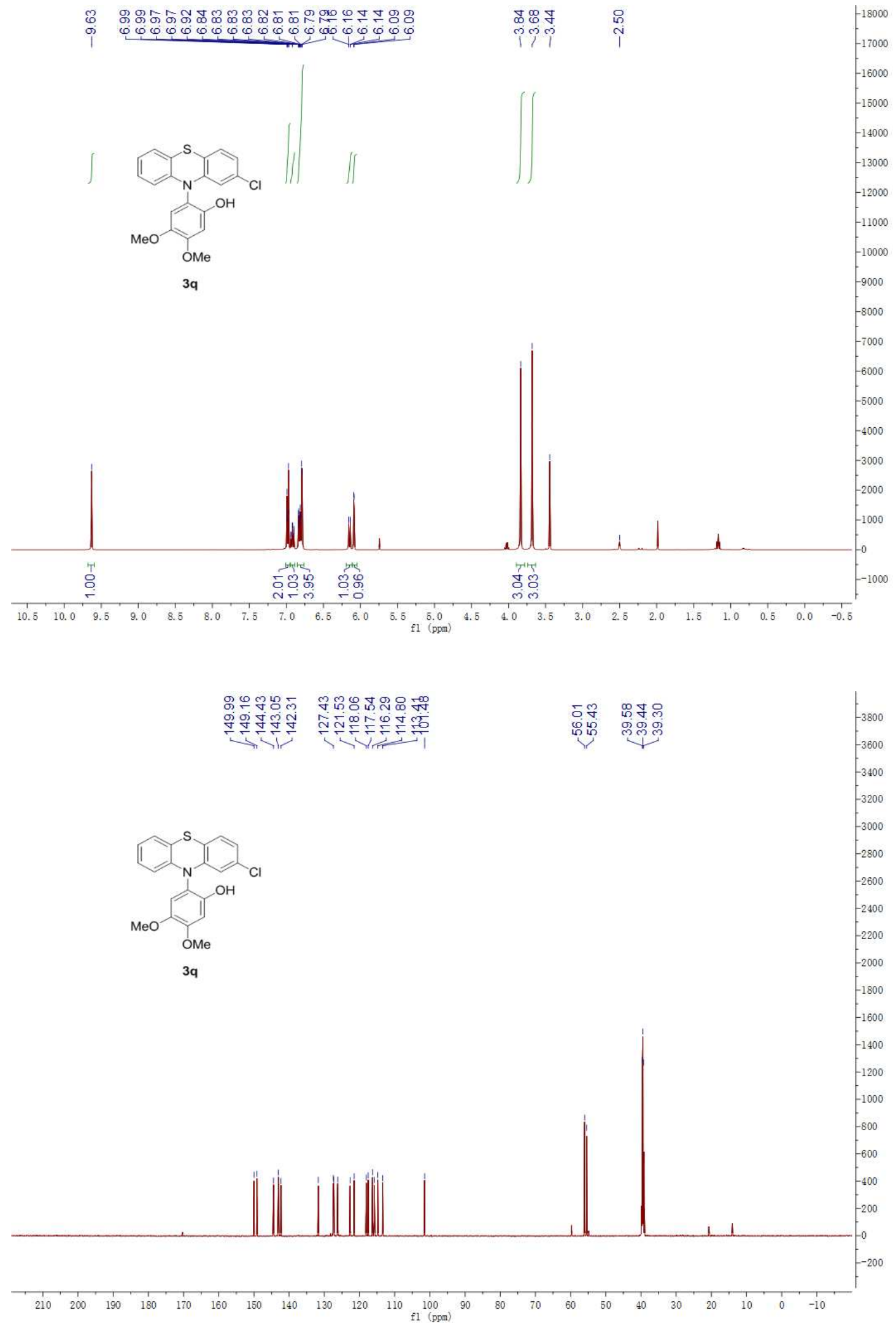



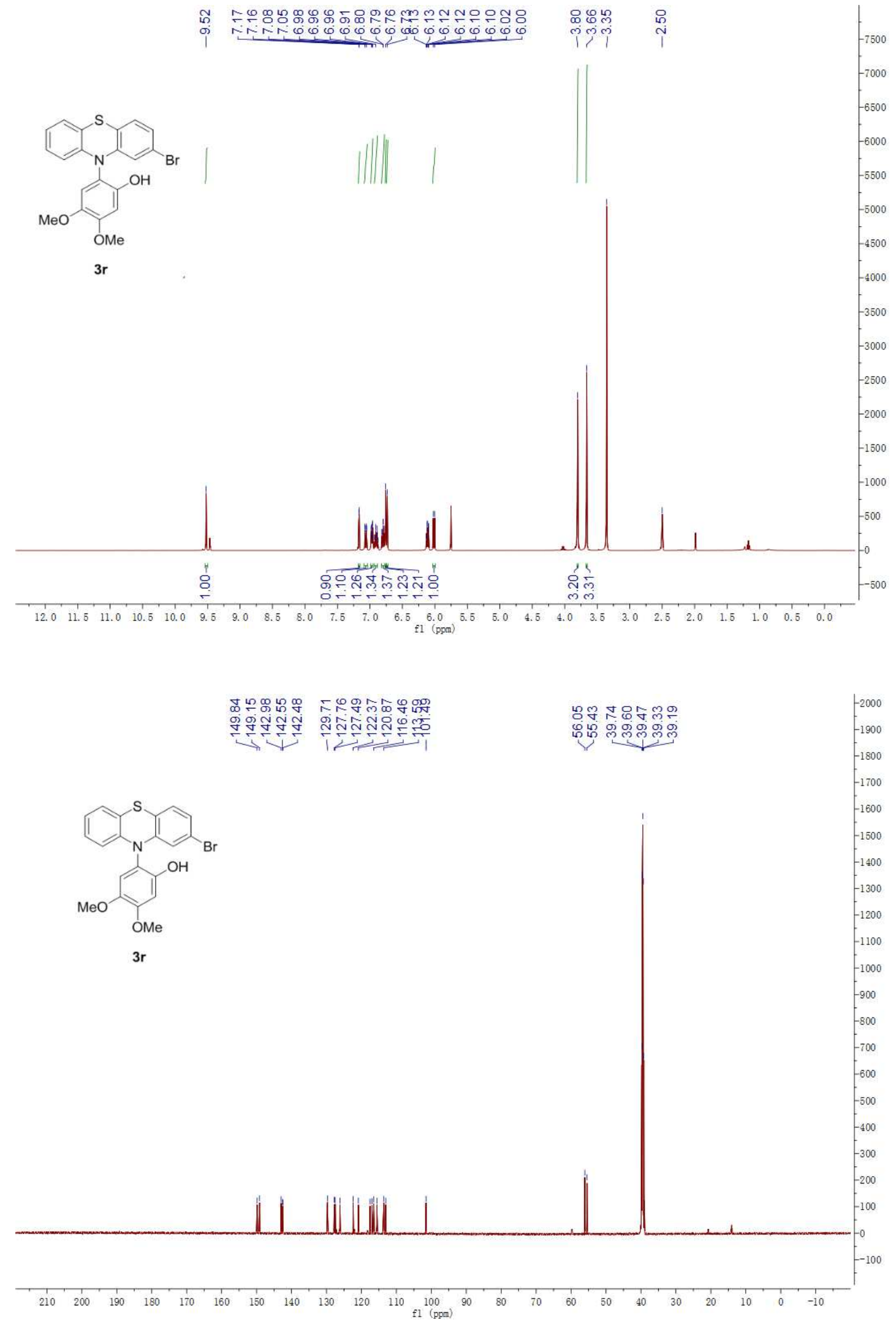

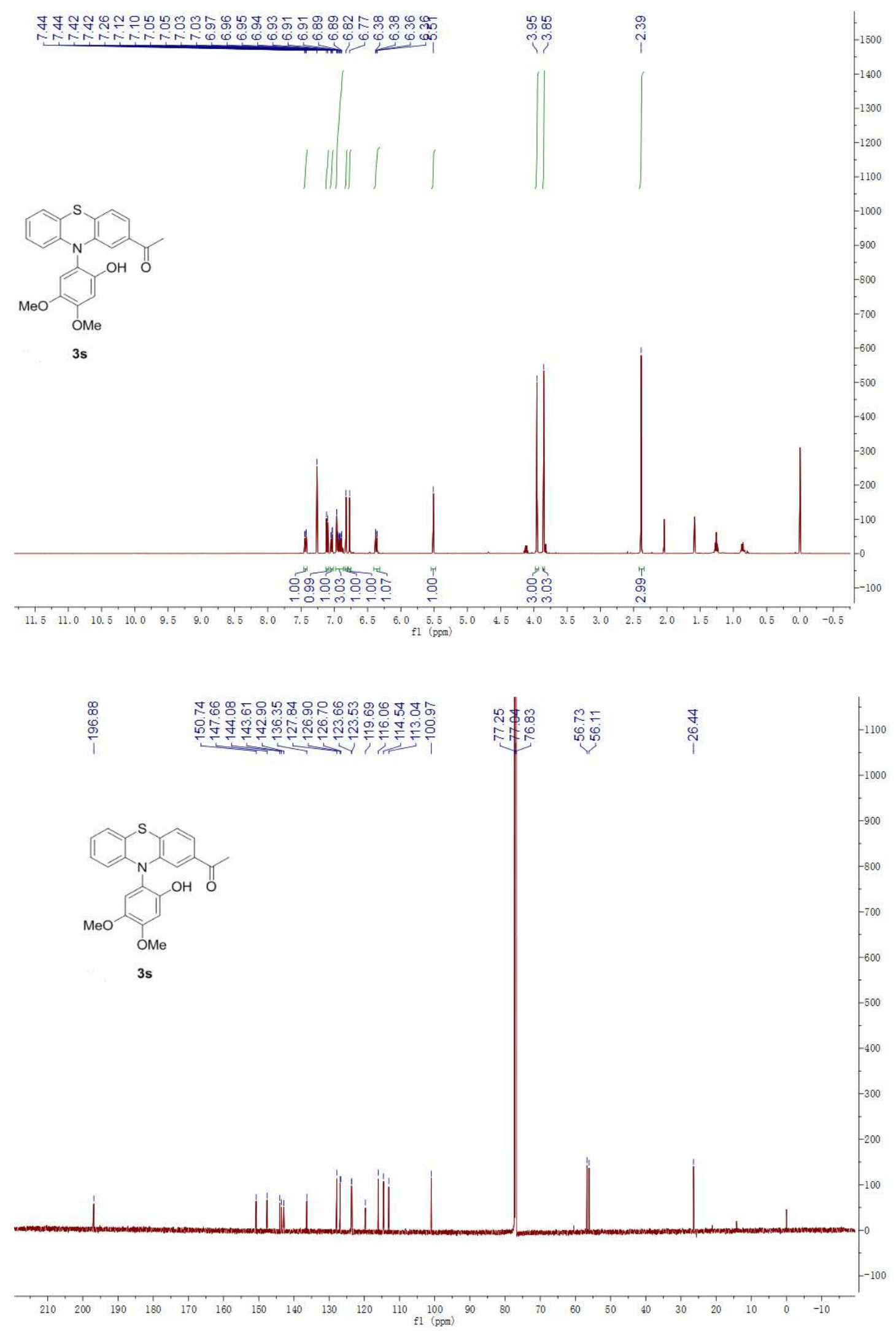

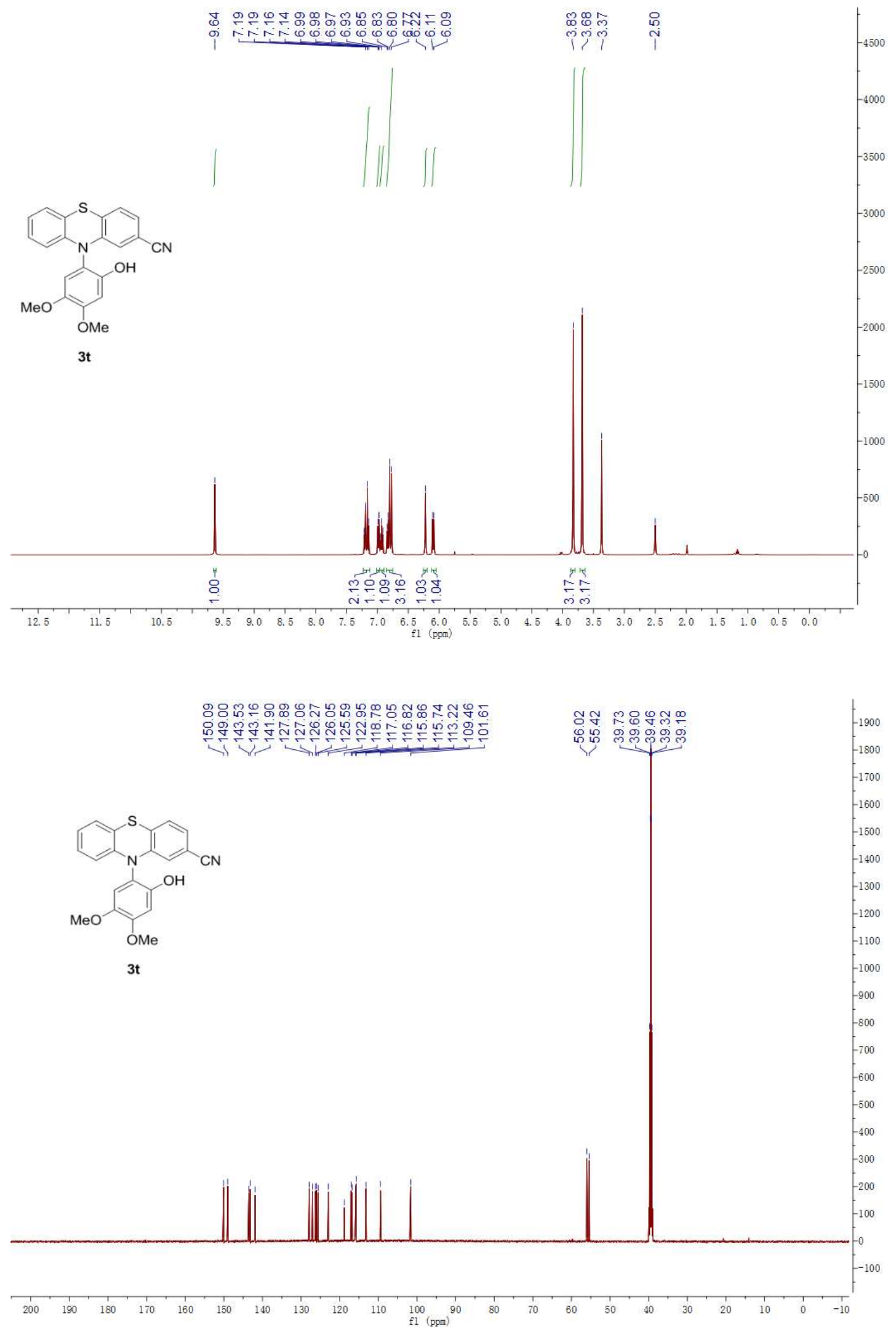


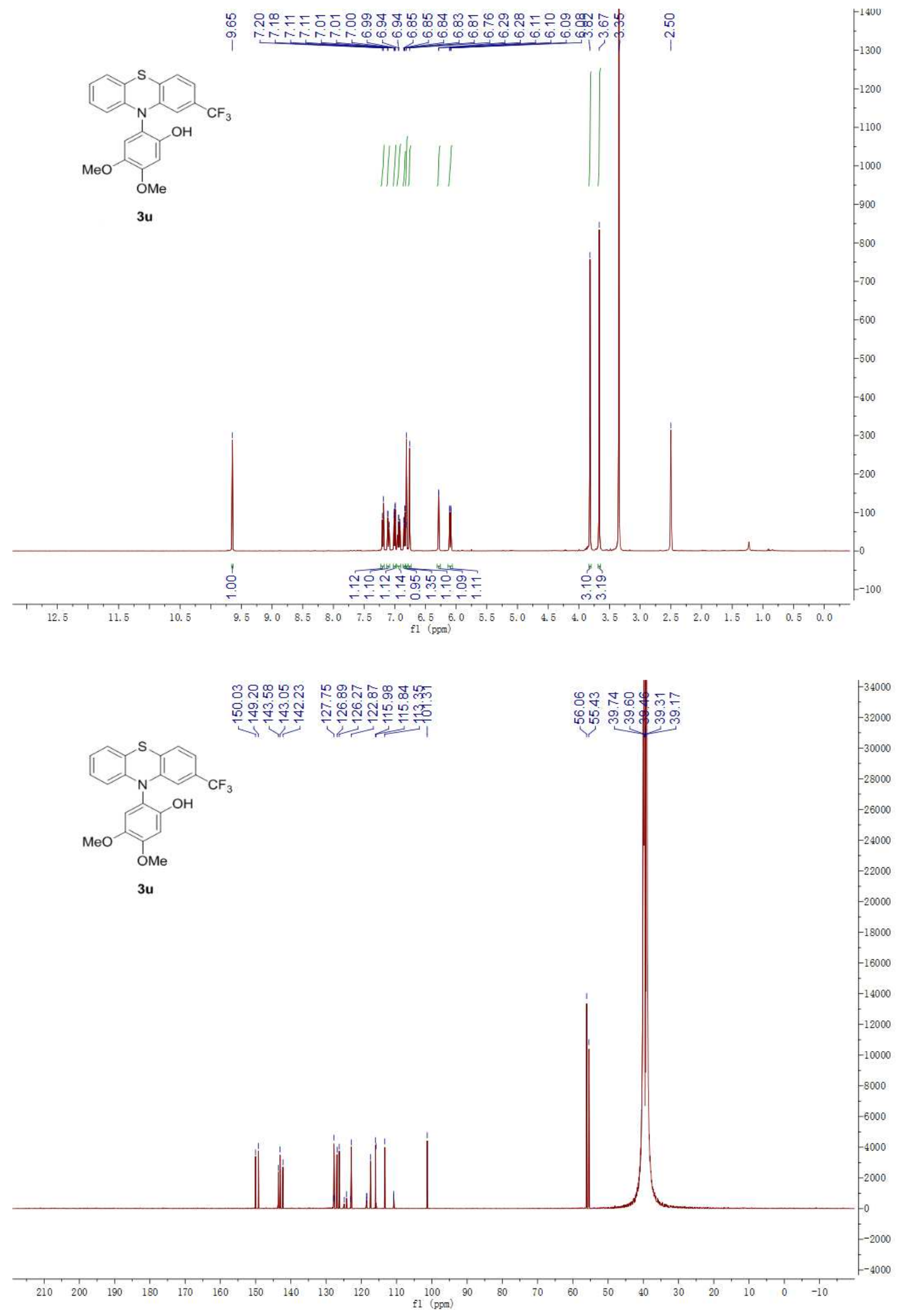



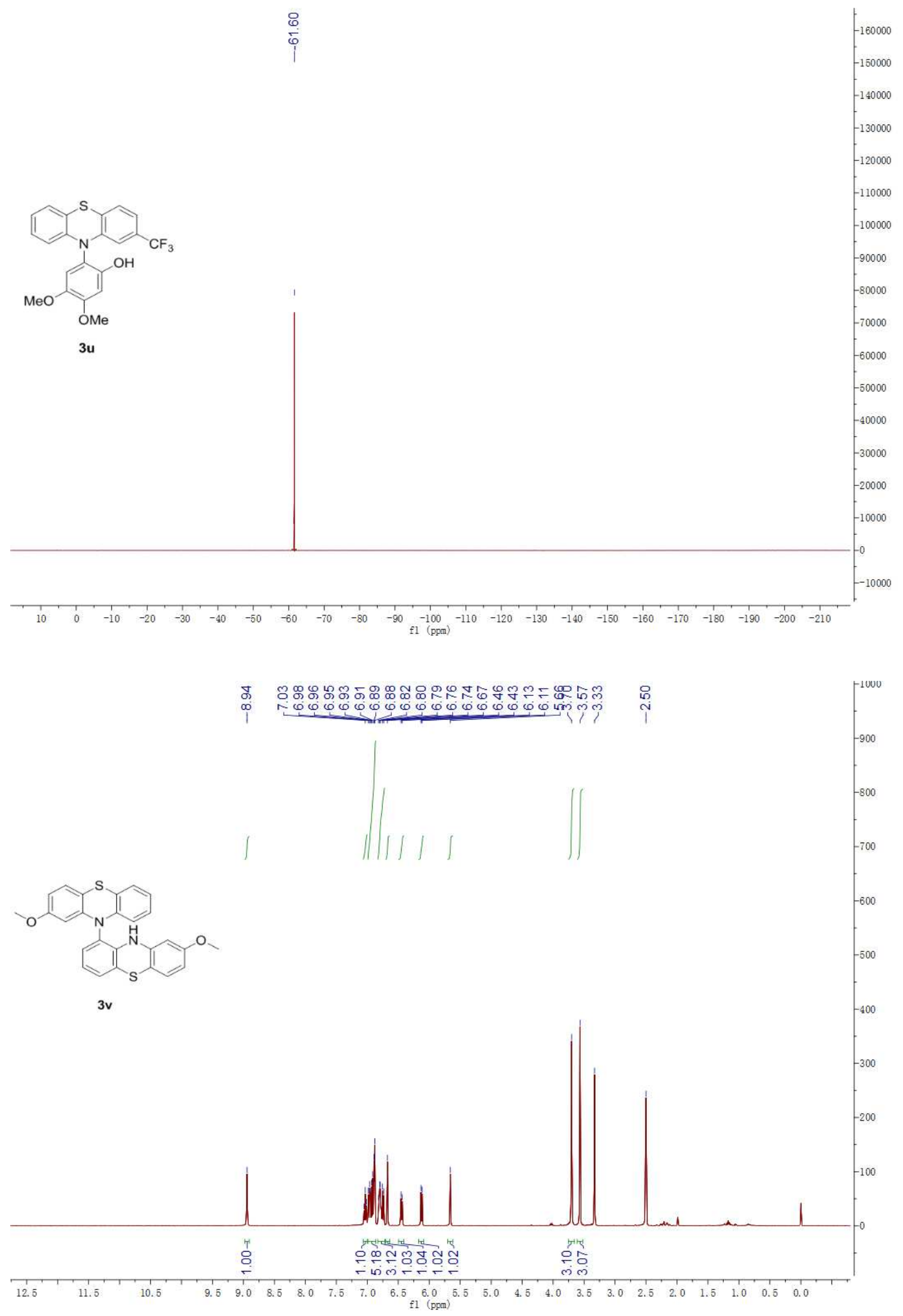


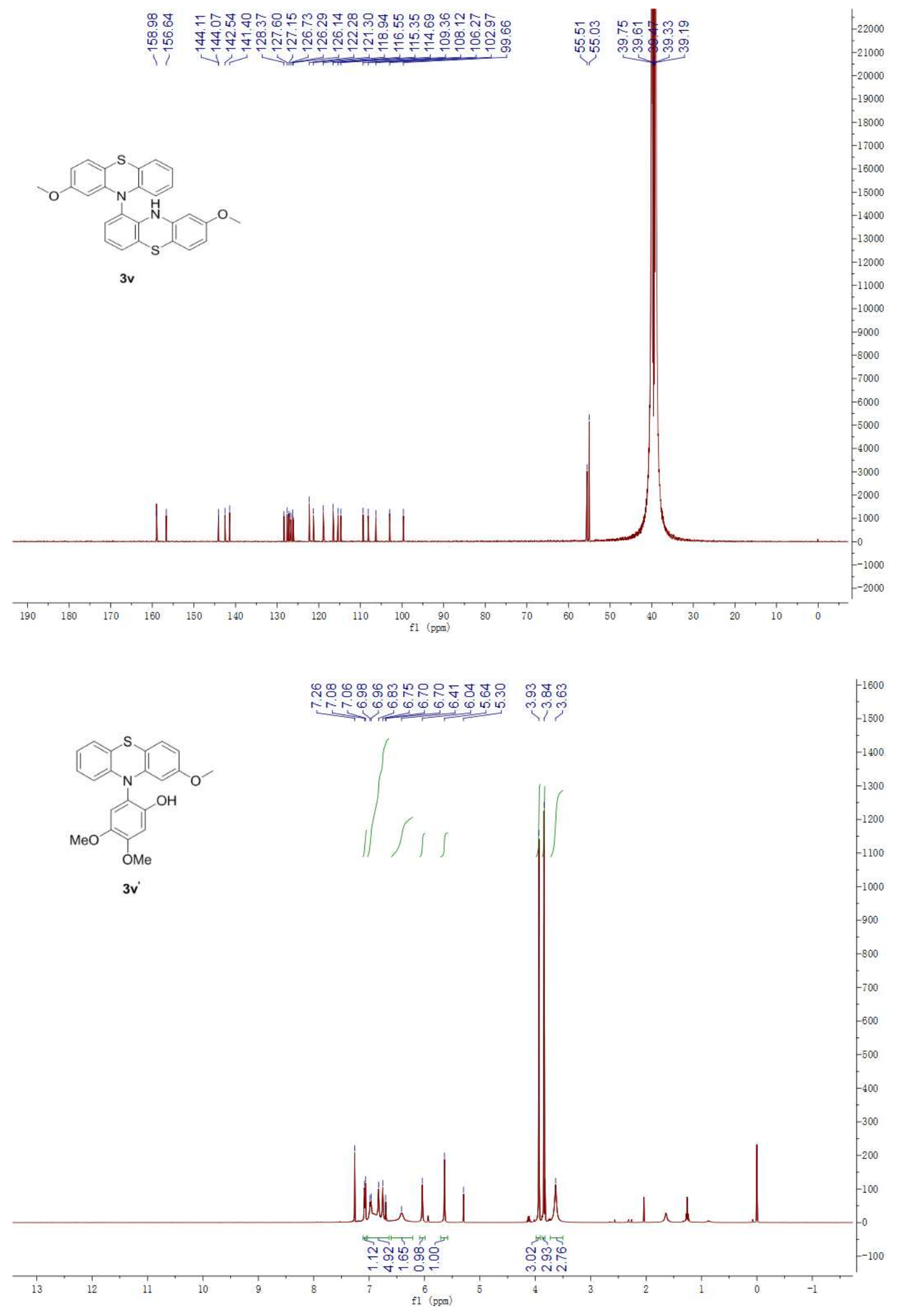



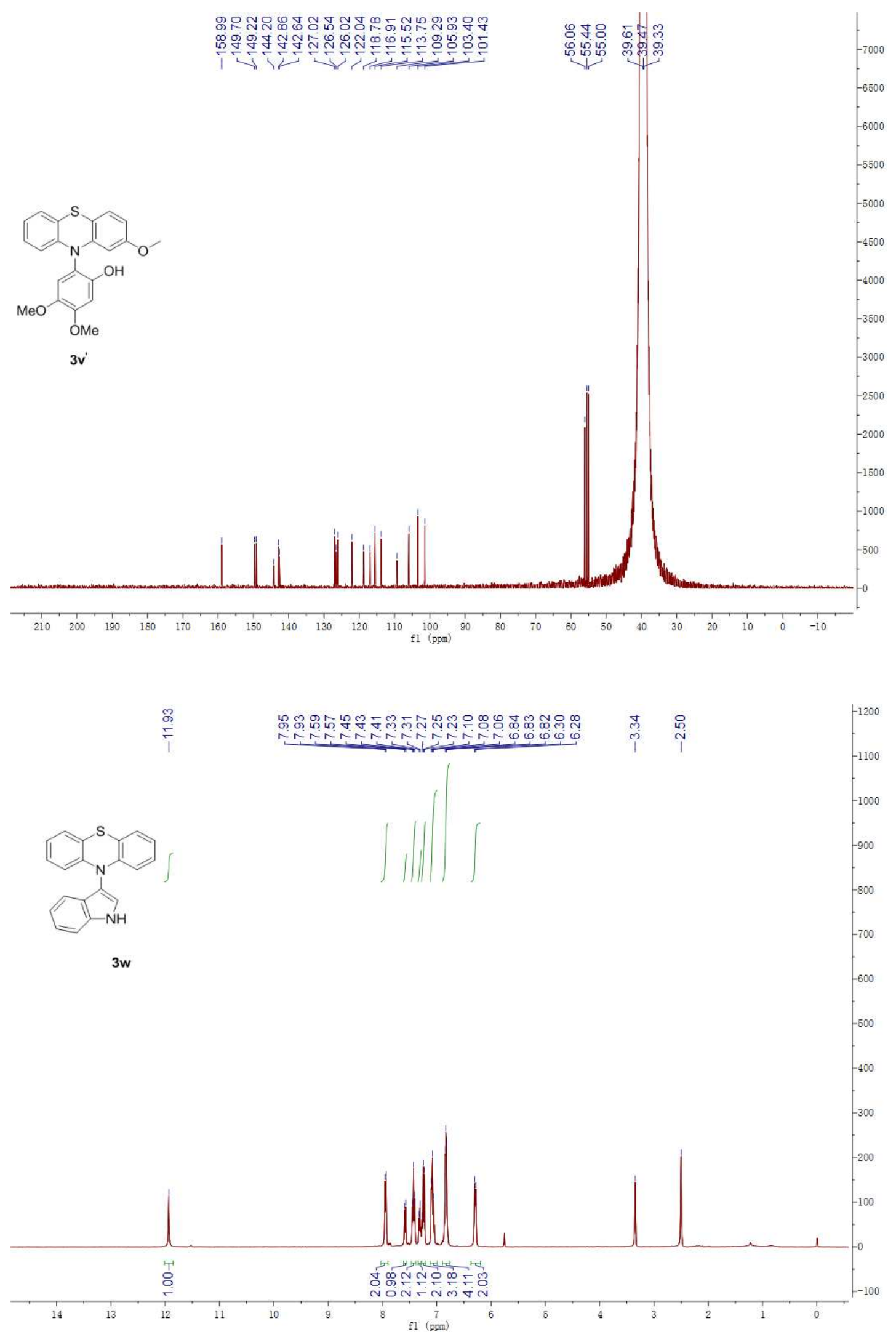


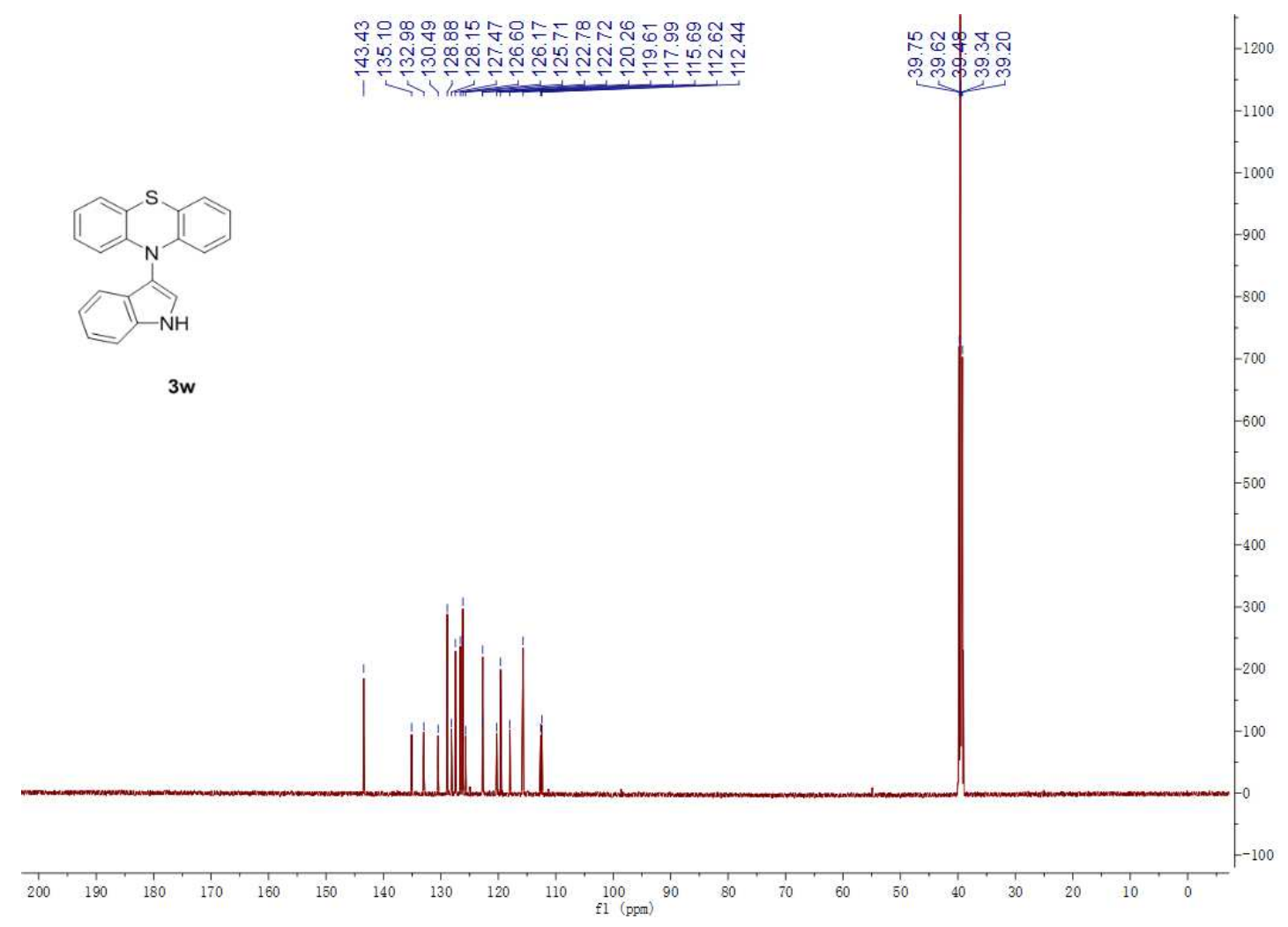

